Pacific

Journal of

Mathematics

\title{
A CLASSIFICATION OF SPHERICAL CONJUGACY CLASSES
}

\section{MAURO COSTANTINI}




\title{
A CLASSIFICATION OF SPHERICAL CONJUGACY CLASSES
}

\author{
MAURO COSTANTINI
}

Let $G$ be a simple algebraic group over an algebraically closed field $k$. We complete the classification of the spherical conjugacy classes of $G$ begun by Carnovale (Pacific J. Math. 245 (2010), 25-45) and the author (Trans. Amer. Math. Soc. 364 (2012), 1997-2019).

\section{Introduction}

Let $G$ be a simple algebraic group over an algebraically closed field $k$. In this paper we complete the classification of the spherical conjugacy classes of $G$ (recalling that a conjugacy class $\mathcal{O}$ in $G$ is called spherical if a Borel subgroup of $G$ has a dense orbit on $\mathcal{O}$ ). There has been a lot of work related to this field, beginning with the work of D. Panyushev [1994; 1999], who classified spherical nilpotent orbits in the Lie algebra of $G$, when the base field is $\mathbb{C}$. R. Fowler and G. Röhrle [2008] classified spherical nilpotent orbits over an algebraically closed field of good characteristic. Then G. Carnovale [2010], exploiting the characterizations of spherical conjugacy classes in terms of the Weyl group given in [Cantarini et al. 2005; Carnovale 2008; 2009], classified the spherical conjugacy classes of $G$ in zero or good, odd characteristic. In [Costantini 2012], we obtained the classification of spherical unipotent conjugacy classes when the characteristic of $k$ is bad, and for characteristic 2 in case $A_{n}$. In the present paper we complete the classification, dealing with nonunipotent conjugacy classes when the characteristic of $k$ is bad, and when $G$ is of type $A_{n}$ and the characteristic is 2 .

The second goal of this paper is the characterization of spherical conjugacy classes in terms of the dimension formula: we prove in Theorem 4.1 that a conjugacy class $\mathcal{O}$ of $G$ is spherical if and only if $\operatorname{dim} \mathcal{O}=\ell\left(w_{\mathcal{O}}\right)+\operatorname{rk}\left(1-w_{\mathcal{O}}\right)$, where $w_{\mathcal{O}}$ is a certain element of the Weyl group attached to $\mathcal{O}$, as defined in the next section. This characterization was obtained over $\mathbb{C}$ in [Cantarini et al. 2005] and in good, odd characteristic in [Carnovale 2008]. An elegant proof was obtained in [Lu 2011] in zero characteristic.

We finally deduce further consequences of the classification.

MSC2010: primary 20E45, 20F55, 20G15; secondary 14M15.

Keywords: spherical conjugacy classes, bad characteristic. 


\section{Preliminaries}

We denote by $\mathbb{C}$ the complex numbers, by $\mathbb{R}$ the reals, and by $\mathbb{Z}$ the integers.

Let $G$ be a simple algebraic group of rank $n$ over $k$, where $k$ is an algebraically closed field. We fix a maximal torus $T$ of $G$, a Borel subgroup $B$ containing $T$, the unipotent radical $U$ of $B$ and the Borel subgroup $B^{-}$opposite to $B$ with unipotent radical $U^{-}$. Then $\Phi$ is the set of roots relative to $T$, and $B$ determines the set of positive roots $\Phi^{+}$and the simple roots $\Delta=\left\{\alpha_{1}, \ldots, \alpha_{n}\right\}$. We write $s_{\alpha}$ for the simple reflection associated to $\alpha \in \Phi$. We use the numbering and the description of the simple roots in terms of the canonical basis $\left(e_{1}, \ldots, e_{k}\right)$ of an appropriate $\mathbb{R}^{k}$ as in [Bourbaki 1981, Planches I-IX]. For the exceptional groups, we write $\beta=\left(m_{1}, \ldots, m_{n}\right)$ for $\beta=m_{1} \alpha_{1}+\cdots+m_{n} \alpha_{n}$. We identify the Weyl group $W$ with $N / T$, where $N$ is the normalizer of $T$. We denote by $w_{0}$ the longest element of $W$. The real space $E=\mathbb{R} \Phi$ is a Euclidean space, endowed with the $W$-invariant scalar product $\left(\alpha_{i}, \alpha_{j}\right)=d_{i} a_{i j}$. Here $\left\{d_{1}, \ldots, d_{n}\right\}$ are relatively prime positive integers such that if $D$ is the diagonal matrix with entries $d_{1}, \ldots, d_{n}$, then $D A$ is symmetric for $A=\left(a_{i j}\right)$ the Cartan matrix.

We put $\Pi=\{1, \ldots, n\}$, and let $\vartheta$ be the symmetry of $\Pi$ induced by $-w_{0}$. We denote by $\ell$ the usual length function on $W$, and by $\operatorname{rk}(1-w)$ the rank of $1-w$ in the geometric representation of $W$.

We use the notation $x_{\alpha}(\xi)$ and $h_{\alpha}(z)$ as in [Steinberg 1968; Carter 1989], for $\alpha \in \Phi, \xi \in k$, and $z \in k^{*}$. For $\alpha \in \Phi$ we put $X_{\alpha}=\left\{x_{\alpha}(\xi) \mid \xi \in k\right\}$, the root-subgroup corresponding to $\alpha$, and $H_{\alpha}=\left\{h_{\alpha}(z) \mid z \in k^{*}\right\}$. Given an element $w \in W$ we denote a representative of $w$ in $N$ by $\dot{w}$. We choose the $x_{\alpha}$ so that, for all $\alpha \in \Phi$, $n_{\alpha}=x_{\alpha}(1) x_{-\alpha}(-1) x_{\alpha}(1)$ lies in $N$ and has image the reflection $s_{\alpha}$ in $W$. Then

$$
\begin{array}{rlrl}
x_{\alpha}(\xi) x_{-\alpha}\left(-\xi^{-1}\right) x_{\alpha}(\xi) & =h_{\alpha}(\xi) n_{\alpha}, & & n_{\alpha}^{2}=h_{\alpha}(-1) \\
n_{\alpha} x_{\alpha}(x) n_{\alpha}^{-1}=x_{-\alpha}(-x), & & h_{\alpha}(\xi) x_{\beta}(x) h_{\alpha}(\xi)^{-1}=x_{\beta}\left(\xi^{\langle\beta, \alpha\rangle} x\right)
\end{array}
$$

for every $\xi \in k^{*}, x \in k$ and $\alpha, \beta \in \Phi$, where $\langle\beta, \alpha\rangle=2(\beta, \alpha) /(\alpha, \alpha)$ [Springer 1998a, Proposition 11.2.1]. The family $\left(x_{\alpha}\right)_{\alpha \in \Phi}$ is called a realization of $\Phi$ in $G$.

We set $T^{w}=\left\{t \in T \mid w t w^{-1}=t\right\}$ and $T_{2}=\left\{t \in T \mid t^{2}=1\right\}$. In particular $T^{w}=T_{2}$ if $w=w_{0}=-1$. We also put $S^{w}=\left\{t \in T \mid w t w^{-1}=t^{-1}\right\}$.

For algebraic groups we use the notation in [Humphreys 1975; Carter 1985]. In particular, for $J \subseteq \Pi$, we have $\Delta_{J}=\left\{\alpha_{j} \mid j \in J\right\}, \Phi_{J}$ is the corresponding root system, $W_{J}$ the Weyl group, $P_{J}$ the standard parabolic subgroup of $G$, and $L_{J}=T\left\langle X_{\alpha} \mid \alpha \in \Phi_{J}\right\rangle$ the standard Levi subgroup of $P_{J}$. For $z \in W$ we put $U_{z}=U \cap z^{-1} U^{-} z$. Then the unipotent radical $R_{u} P_{J}$ of $P_{J}$ is $U_{w_{0} w_{J}}$, where $w_{J}$ is the longest element of $W_{J}$. Moreover $U \cap L_{J}=U_{w_{J}}$ is a maximal unipotent subgroup of $L_{J}$ (of dimension $\ell\left(w_{J}\right)$ ), and $T_{J}=T \cap L_{J}^{\prime}$ is a maximal torus of $L_{J}^{\prime}$. For unipotent classes in exceptional groups we use the notation in [Carter 1985; 
Spaltenstein 1982]. We use the description of centralizers of involutions as in [Iwahori 1970].

If $X$ is a $G$-variety and $x \in X$, we denote by $G . x$ the $G$-orbit of $x$ and by $G_{x}$ the isotropy subgroup of $x$ in $G$. We say that $X$ is spherical if a Borel subgroup of $G$ has a dense orbit on $X$. It is well known (see [Brion 1986; Vinberg 1986] in characteristic 0, [Grosshans 1992; Knop 1995] in positive characteristic) that $X$ is spherical if and only if the set $\mathcal{V}$ of $B$-orbits in $X$ is finite. If $H$ is a closed subgroup of $G$ and the homogeneous space $G / H$ is spherical, we say that $H$ is a spherical subgroup of $G$.

Let $g$ be an element of $G$ with Jordan decomposition $g=s u$, for $s$ semisimple and $u$ unipotent. Using a terminology slightly different from the usual, we say that $g$ is mixed if $s \notin Z(G)$ and $u \neq 1$. For each conjugacy class $\mathcal{O}$ in $G, w=w_{\mathcal{O}}$ is the unique element of $W$ such that $B w B \cap \mathcal{O}$ is open dense in $\mathcal{O}$.

If $x$ is an element of a group $K$ and $H \leq K$, we denote by $C(x)$ the centralizer of $x$ in $K$, and by $C_{H}(x)$ the centralizer of $x$ in $H$. If $x, y \in K$, then $x \sim y$ means that $x$ and $y$ are conjugate in $K$.

If $H$ is an algebraic group, we denote by $B(H)$ a Borel subgroup of $H$. We denote the identity matrix of order $r$ by $I_{r}$. Finally, in the remainder of the paper we denote by $p$ the characteristic of $k$ (hence $p$ may be 0 ).

\section{The classification}

We recall that the bad primes for the individual types of simple groups are as follows:

- none when $G$ has type $A_{n}$;

- $p=2$ when $G$ has type $B_{n}, C_{n}$ or $D_{n}$;

- $p=2$ or 3 when $G$ has type $G_{2}, F_{4}, E_{6}$ or $E_{7}$;

- $p=2,3$ or 5 when $G$ has type $E_{8}$.

For convenience we assume $G$ simply connected, so that centralizers of semisimple elements are connected [Carter 1985, Theorem 3.5.6]. However the classification of spherical conjugacy classes in $G$ is independent of the isogeny class. More precisely, let $D \leq Z(G)$ and $\bar{G}=G / D$. For the canonical projection $\pi: G \rightarrow \bar{G}$ and $g \in G$, put $\bar{g}=\pi(g)$. Then it is clear that the conjugacy class of $\bar{g}$ in $\bar{G}$ is spherical if and only if the conjugacy class of $g$ in $G$ is spherical; see also the discussion at the beginning of [Costantini 2010, §6].

We put $\widetilde{\Pi}=\Pi \cup\{0\}$ and $\widetilde{\Delta}=\Delta \cup\left\{\alpha_{0}\right\}$, where $\alpha_{0}=-\beta$ for the highest root $\beta$ of $\Phi^{+}$. Thus $\widetilde{\Pi}$ labels the vertices of the extended Dynkin diagram of the root system $\Phi$. For $J \subset \widetilde{\Pi}$, let $\Phi_{J}=\mathbb{Z}\left\{\alpha_{i} \mid i \in J\right\} \cap \Phi$ and

$$
L_{J}=\left\langle T, X_{\alpha} \mid \alpha \in \Phi_{J}\right\rangle \text {. }
$$


This is called a pseudo-Levi subgroup of $G$ (in the sense of [Sommers 1998]). Then the following holds:

Proposition 3.1 [McNinch and Sommers 2003, Propositions 30 and 32]. Let $t$ in $G$ be semisimple. Then $C(t)$ is conjugate to a subgroup $L_{J}$ for some $J \subset \widetilde{\Pi}$.

Suppose that the characteristic of $k$ is good for $G$. Let $J \subset \widetilde{\Pi}$. Then there is $t \in G$ such that $L_{J}=C(t)$.

We recall some basic facts which have been proved for zero or good, odd characteristic.

Theorem 3.2. Let $p \neq 2$, and let $\mathcal{O}$ be a spherical conjugacy class of a connected reductive algebraic group. If $\mathcal{O} \cap B w B$ is nonempty, then $w^{2}=1$.

Proof. If $p$ is zero or good and odd then this is [Carnovale 2008, Theorem 2.7]. The same proof holds as long as $p \neq 2$; see also [Carnovale and Costantini 2013, Theorem 2.1].

Remark 3.3. Let $M(W)$ denote the Richardson-Springer monoid, i.e., the monoid generated by the symbols $r_{\alpha}$ for $\alpha \in \Delta$, subject to the braid relations and the relation $r_{\alpha}^{2}=r_{\alpha}$ for $\alpha \in \Delta$. Given a spherical $G$-variety, there is an $M(W)$-action on the set $\mathcal{V}$ of its $B$-orbits. Under additional conditions, one can also define an action of $W$ on $\mathcal{V}$. These actions have been introduced in [Richardson and Springer 1990] and [Knop 1995], respectively, and they have been further analyzed and applied in [Brion 2001; Mars and Springer 1998, §4.1; Springer 1998b]. The actions of $M(W)$ and $W$ have been used to prove [Carnovale 2008, Theorem 2.7]. By [Knop 1995, Theorem 4.2(b)], a case in which the action of $W$ is defined is when $p \neq 2$. This allows one to extend the proof of [Carnovale 2008, Theorem 2.7] to the case $p \neq 2$, as done in [Carnovale and Costantini 2013, Theorem 2.1]. We shall come back to this point after the achievement of the classification of spherical conjugacy classes in characteristic 2 .

Let $\mathcal{O}$ be a conjugacy class of $G$ and let $\mathcal{V}$ be the set of $B$-orbits in $\mathcal{O}$. There is a natural map $\phi: \mathcal{V} \rightarrow W$ associating to $v \in \mathcal{V}$ the element $w$ in the Weyl group of $G$ for which $v \subseteq B w B$ (equivalently, for which $v \cap B w B \neq \varnothing$ ).

Theorem 3.4. Let $p \neq 2$, and let $\mathcal{O}$ be a conjugacy class in a connected reductive algebraic group. If $\operatorname{Im}(\phi)$ contains only involutions in $W$, then $\mathcal{O}$ is spherical.

Proof. If $p$ is zero, or good and odd this is [Carnovale 2009, Theorem 5.7]. The same proof holds as long as $p \neq 2$, once it is noticed again that the action of $W$ on $\mathcal{V}$ is defined.

Theorem 3.5 [Cantarini et al. 2005, Theorem 25; Carnovale 2008, Theorem 4.4]. A class $\mathcal{O}$ in a connected reductive algebraic group $G$ over an algebraically closed field of zero or good odd characteristic is spherical if and only if there exists $v$ in $\mathcal{V}$ 
such that $\ell(\phi(v))+\operatorname{rk}(1-\phi(v))=\operatorname{dim} \mathcal{O}$. If this is the case, $v$ is the dense $B$-orbit in $\mathcal{O}$ and $\phi(v)=w_{\mathcal{O}}\left(\right.$ and $\left.v=\mathcal{O} \cap B w_{\mathcal{O}} B\right)$.

For any conjugacy class $\mathcal{O}$, the element $w_{\mathcal{O}}$ of the Weyl group is an involution, i.e., $w_{\mathcal{O}}^{2}=1$, is the unique maximal element in its conjugacy class and is of the form $w_{\mathcal{O}}=w_{0} w_{J}$, for a certain $\vartheta$-invariant subset $J$ of $\Pi$ such that $w_{0}(\alpha)=w_{J}(\alpha)$ for every $\alpha \in \Delta_{J}$ [Carnovale 2008, Lemma 3.5; Chan et al. 2010, Corollary 2.11; Perkins and Rowley 2002].

We indicate the strategy we followed to determine the classification. Let $G_{\mathbb{C}}$ be the corresponding group over $\mathbb{C}$. We have shown in [Cantarini et al. 2005] that for every spherical conjugacy class $\mathcal{C}$ of $G_{\mathbb{C}}$ there exists an involution $w=w(\mathcal{C})$ in $W$ such that $\operatorname{dim} \mathcal{C}=\ell(w)+\operatorname{rk}(1-w)$, with $\mathcal{C} \cap B w B \neq \varnothing$ (in fact even $\left.\mathcal{C} \cap B w B \cap B^{-} \neq \varnothing\right)$. For each group $G$ we introduce a certain set $\mathcal{O}(G)$ of semisimple or mixed conjugacy classes; this set is suggested by the classification in characteristic zero. For each $\mathcal{O} \in \mathcal{O}(G)$ there is a certain spherical conjugacy class $\mathcal{C}$ in $G_{\mathbb{C}}$ such that $\operatorname{dim} \mathcal{O}=\operatorname{dim} \mathcal{C}$. Let $w=w_{\mathcal{C}}$. Our aim is to show that $\mathcal{O} \cap B w B \neq \varnothing$, so that $\mathcal{O}$ is in fact spherical by the following proposition. Finally we show that any conjugacy class not in $\mathcal{O}(G)$ is not spherical.

For convenience of the reader we shall give tables for the nonunipotent spherical conjugacy classes. In the tables we give a representative $g$ of the spherical conjugacy class $\mathcal{O}$, the subset $J$ of $\Pi$ for which $w_{\mathcal{O}}=w_{0} w_{J}$, the decomposition of $w_{\mathcal{O}}$ into the product of orthogonal reflections, the type of $C(g)$ when $g$ is semisimple and the dimension of $\mathcal{O}$.

We recall the following result, proved in [Cantarini et al. 2005, Theorem 5] over $\mathbb{C}$, but which is valid with the same proof over any algebraically closed field.

Proposition 3.6. Suppose that $\mathcal{O}$ contains an element $x \in B w B$. Then

$$
\operatorname{dim} B \cdot x \geq \ell(w)+\operatorname{rk}(1-w) .
$$

In particular, $\operatorname{dim} \mathcal{O} \geq \ell(w)+\operatorname{rk}(1-w)$. If in addition $\operatorname{dim} \mathcal{O} \leq \ell(w)+\operatorname{rk}(1-w)$, then $\mathcal{O}$ is spherical, $w=w_{\mathcal{O}}$ and $B . x$ is the dense $B$-orbit in $\mathcal{O}$.

If $g$ is in $Z(G)$, then $g \in T, \mathcal{O}_{g}=\{g\}$ and $w_{\mathcal{O}}=1$. In the remainder of the paper we consider only noncentral conjugacy classes.

We shall use the following result.

Lemma 3.7. Assume the positive roots $\beta_{1}, \ldots, \beta_{\ell}$ are such that $\left[X_{ \pm \beta_{i}}, X_{ \pm \beta_{j}}\right]=1$ for every $1 \leq i<j \leq \ell$. Then, for $g=n_{\beta_{1}} \cdots n_{\beta_{\ell}} x_{\beta_{1}}(1) \cdots x_{\beta_{\ell}}(1)$ and $h \in T$ such that $\beta_{i}(h) \neq 1$ for $i=1, \ldots, \ell$, we have

$$
g h g^{-1} \in B w B \cap B^{-}
$$

where $w=s_{\beta_{1}} \cdots s_{\beta_{\ell}}$. 
Proof. By [Carter 1989, p. 106], for every positive root $\alpha$ and every $t \in k^{*}$ we have $x_{-\alpha}(t)=x_{\alpha}\left(t^{-1}\right) n_{\alpha} x_{\alpha}\left(t^{-1}\right) h^{\prime}$ for a certain $h^{\prime} \in T$, so that $x_{-\alpha}(t) \in B s_{\alpha} B \cap B^{-}$. Hence, for every $i=1, \ldots, \ell$, by (2-1) we have

$$
\begin{aligned}
n_{\beta_{i}} x_{\beta_{i}}(1) h\left(n_{\beta_{i}} x_{\beta_{i}}(1)\right)^{-1} & =n_{\beta_{i}} x_{\beta_{i}}(1) h x_{\beta_{i}}(-1) h^{-1} h n_{\beta_{i}}^{-1} \\
& =n_{\beta_{i}} x_{\beta_{i}}\left(1-\beta_{i}(h)\right) n_{\beta_{i}}^{-1} n_{\beta_{i}} h n_{\beta_{i}}^{-1} \\
& =x_{-\beta_{i}}\left(\beta_{i}(h)-1\right) h_{i} \\
& \in B s_{\beta_{i}} B \cap B^{-}
\end{aligned}
$$

where $h_{i}=n_{\beta_{i}} h n_{\beta_{i}}^{-1} \in T$. Let $t_{1}, \ldots, t_{\ell} \in k^{*}$. Then

$$
\left(x_{\beta_{1}}\left(t_{1}^{-1}\right) \cdots x_{\beta_{\ell}}\left(t_{\ell}^{-1}\right)\right)^{-1} x_{-\beta_{1}}\left(t_{1}\right) \cdots x_{-\beta_{\ell}}\left(t_{\ell}\right)\left(x_{\beta_{1}}\left(t_{1}^{-1}\right) \cdots x_{\beta_{\ell}}\left(t_{\ell}^{-1}\right)\right)
$$

lies in $n_{\beta_{1}} X_{\beta_{1}} \cdots n_{\beta_{\ell}} X_{\beta_{\ell}} T=n_{\beta_{1}} \cdots n_{\beta_{\ell}} X_{\beta_{1}} \cdots X_{\beta_{\ell}} T \subseteq w B$. Therefore

$$
g h g^{-1}=x_{-\beta_{1}}\left(\beta_{1}(h)-1\right) \cdots x_{-\beta_{\ell}}\left(\beta_{\ell}(h)-1\right) h_{1} \cdots h_{\ell} \in B w B \cap B^{-} .
$$

The hypothesis of the lemma is satisfied for instance if $\beta_{1}, \ldots, \beta_{\ell}$ are pairwise orthogonal and long, as in [Costantini 2010, Lemma 4.1]. In characteristic 2, we have $\left[X_{\gamma}, X_{\delta}\right]=1$ for every pair $(\gamma, \delta)$ of orthogonal roots.

Let $\mathcal{O}$ be the conjugacy class of $x \in G$. In general the orbit map $\pi: G / C(x) \rightarrow \mathcal{O}$ is a bijective morphism, which may not be separable (i.e., an isomorphism). Nevertheless, we have the following result:

Lemma 3.8 [Fowler and Röhrle 2008, Remark 2.14]. Let $\mathcal{O}$ be a $G$-orbit with isotropy subgroup $H$. Then $\mathcal{O}$ is spherical if and only if $G / H$ is spherical.

Proposition 3.9. Let $g \in G$ with Jordan decomposition $g=s u$ for $s$ semisimple and $u$ unipotent. If $\mathcal{O}_{g}$ is spherical then $\mathcal{O}_{s}$ and $\mathcal{O}_{u}$ are spherical.

Proof. By Lemma 3.8, $C(\mathrm{~g})=C(s) \cap C(u)$ is a spherical subgroup of $G$. Hence both $C(s)$ and $C(u)$ are spherical subgroups of $G$ and, by Lemma 3.8, $\mathcal{O}_{s}$ and $\mathcal{O}_{u}$ are spherical.

For $J \subseteq \Pi$ we put $T_{J}=T \cap L_{J}^{\prime}$, a maximal torus of the derived subgroup $L_{J}^{\prime}$ of the standard Levi subgroup $L_{J}$, so that $B_{J}=T_{J} U_{w_{J}}$ is a Borel subgroup of $L_{J}^{\prime}$.

Lemma 3.10. Let $\mathcal{O}$ be a conjugacy class of $G$ and $\mathcal{F} \subseteq \mathcal{O}$. Assume there exists $J \subseteq \Pi$ such that $\mathcal{F} \subseteq L_{J}$ and $\left(B_{J} \cdot x\right)_{x \in \mathcal{F}}$ is a family of pairwise distinct $B_{J}$-orbits. Then the family $(B . x)_{x \in \mathcal{F}}$ consists of pairwise distinct $B$-orbits.

Proof. Let $x$ and $y$ be elements of $\mathcal{F}$, and assume B.x B.y. Then there exists $b \in B$ such that $b x b^{-1}=y$, i.e., $b x=y b$. Since $B=T U_{w_{J}} U_{w_{0} w_{J}}$, where $U_{w_{0} w_{J}}$ is the unipotent radical of the standard parabolic subgroup $P_{J}$, we can write $b=t u_{1} u_{2}$ with $t \in T, u_{1} \in U_{w_{J}}$ and $u_{2} \in U_{w_{0} w_{J}}$, so that $t u_{1} u_{2} x=y t u_{1} u_{2}$. Since $U_{w_{0} w_{J}}$ is normal in $P_{J}$, from uniqueness of expression we get $t u_{1} x=y t u_{1}$. We may 
decompose $T=T_{J} S$ where $S=\left(\bigcap_{i \in J} \operatorname{ker} \alpha_{i}\right)^{\circ}$, and $t=t_{1} t_{2}$ with $t_{1} \in T_{J}, t_{2} \in S$. Then $S \leq C\left(L_{J}\right)$, so that $t_{1} u_{1} x=y t_{1} u_{1}$. But $t_{1} u_{1}$ lies in $B_{J}$, and we conclude that $B_{J} \cdot x=B_{J} \cdot y$. Therefore $x=y$ and we are done.

Lemma 3.11. Let $x$ be a semisimple element of $G$ with $C(x)=L_{J}$, a pseudo-Levi subgroup of $G$, and assume $\mathcal{O}_{x}$ is spherical. Let $\tilde{x}$ be a semisimple element in $G_{\mathbb{C}}$ such that $C(\tilde{x})=L_{J}\left(\right.$ in $\left.G_{\mathbb{C}}\right)$. Then $\mathcal{O}_{\tilde{x}}$ is spherical.

Proof. First we note that such an $\tilde{x}$ exists, by Proposition 3.1. By Lemma 3.8 and [Brundan 1998, Theorem 2.2(i)], it follows that $\mathcal{O}_{\tilde{x}}$ is a spherical semisimple conjugacy class in $G_{\mathbb{C}}$.

Type $A_{n}, \boldsymbol{n} \geq 1$. For every $i=1, \ldots,\left[\frac{1}{2}(n+1)\right]$, we denote the root $e_{i}-e_{n+2-i}$ by $\beta_{i}$.

Proposition 3.12. Let $G=\mathrm{SL}(2)$, any characteristic. Let $\mathcal{O}$ be a conjugacy class of $G$. Then $\mathcal{O} \cap B w_{\mathcal{O}} B \cap B^{-}$is nonempty, $\operatorname{dim} \mathcal{O}=\ell\left(w_{\mathcal{O}}\right)+r k\left(1-w_{\mathcal{O}}\right)$ and $\mathcal{O}$ is spherical.

Proof. We may work (and usually do) up to a central element, hence we may assume $\mathcal{O}=\mathcal{O}_{x}, x$ either unipotent or semisimple. If $x$ is unipotent then the result follows from [Cantarini et al. 2005, Proposition 11], whose proof is characteristic-free. If $x$ is semisimple, then either $x$ is central, or $x$ is regular. In the first case $C(x)=G$, and in the second case we may assume $C(x)=T$. Now

$$
x=\left(\begin{array}{cc}
f & 0 \\
0 & 1 / f
\end{array}\right)
$$

for a certain $f \neq \pm 1$. Let

$$
g=\left(\begin{array}{rr}
0 & 1 \\
-1 & 0
\end{array}\right)\left(\begin{array}{ll}
1 & 1 \\
0 & 1
\end{array}\right)
$$

Then $g x g^{-1} \in \mathcal{O} \cap B w B \cap B^{-}$by Lemma 3.7, where $w=w_{0}$, with $\operatorname{dim} \mathcal{O}=2=$ $\ell\left(w_{0}\right)+\operatorname{rk}\left(1-w_{0}\right)$. We conclude by Proposition 3.6.

Lemma 3.13. Let $H$ be connected and reductive, any characteristic. Then $H$ has a regular spherical conjugacy class if and only if the semisimple part of $H$ is of type $A_{1}^{r}$. In this case every conjugacy class is spherical.

Proof. Without loss of generality we may assume $H=Z \times G_{1} \times \cdots \times G_{r}$, where $Z=Z(H)^{\circ}$ and $G_{i}$ is simple for each $i=1, \ldots, r$. Let $n_{i}=\operatorname{rk} G_{i}$ and $N_{i}$ the number of positive roots of $G_{i}$ for $i=1, \ldots, r$. Let $x=\left(z, x_{1}, \ldots, x_{r}\right)$ be an element of $H$ and $\mathcal{O}=\mathcal{O}_{x}$. Then $\mathcal{O}$ is spherical if and only if each $G_{i} . x_{i}$ is spherical in $G_{i}$, and $x$ is regular if and only if each $x_{i}$ is regular in $G_{i}$. Moreover, a spherical $G_{i}$-conjugacy class in $G_{i}$ has dimension at most $n_{i}+N_{i}$, while $G_{i} . x_{i}$ is regular in $G_{i}$ if and only if its dimension is $2 N_{i}$. 
If the semisimple part of $H$ is of type $A_{1}^{r}$, then every conjugacy class of $H$ is spherical by Proposition 3.12.

Suppose there exists a regular spherical conjugacy class. Then $2 N_{i} \leq n_{i}+N_{i}$ for every $i$, which is possible if and only if $N_{i}=n_{i}=1$ for every $i$. Hence the semisimple part of $H$ is of type $A_{1}^{r}$.

Lemma 3.14. Let $H=G L(3)$, any characteristic, $g$ a regular element of $H$. Then there exists a subset $\mathcal{F}=\left\{x_{m} \mid m \in k^{*}\right\}$ of $\mathcal{O}_{g}$ such that $\left(B(H) x_{m}\right)_{m \in k^{*}}$ consists of pairwise distinct $B(H)$-orbits.

Proof. For $m, a, b, c \in k^{*}$, let

$$
\begin{aligned}
x_{m} & =x_{m}(a, b, c)=\left(\begin{array}{ccc}
0 & 0 & \frac{a b c}{m} \\
0 & -m & -\frac{(a+m)(b+m)(c+m)}{m} \\
1 & 1 & a+b+c+m
\end{array}\right) \\
& =\left(\begin{array}{rrr}
0 & 0 & 1 \\
0 & -1 & 0 \\
1 & 0 & 0
\end{array}\right)\left(\begin{array}{rrc}
1 & 0 & 0 \\
0 & m & 0 \\
0 & 0 & \frac{a b c}{m}
\end{array}\right)\left(\begin{array}{ccc}
1 & 1 & a+b+c+m \\
0 & 1 & \frac{(a+m)(b+m)(c+m)}{m^{2}} \\
0 & 0 & 1
\end{array}\right) \in w_{0} B .
\end{aligned}
$$

From the uniqueness of Bruhat decomposition, we have B. $x_{m} \cap w_{0} B=T . x_{m}$; moreover, $C_{T}\left(x_{m}\right)$ consists of scalar matrices, and

$$
S=\left\{\left(\begin{array}{ccc}
\alpha & 0 & 0 \\
0 & \beta & 0 \\
0 & 0 & 1
\end{array}\right) \mid \alpha, \beta \in k^{*}\right\}
$$

acts as

$$
\left(\begin{array}{lll}
\alpha & 0 & 0 \\
0 & \beta & 0 \\
0 & 0 & 1
\end{array}\right) \cdot x_{m}=\left(\begin{array}{ccc}
0 & 0 & \alpha \frac{a b c}{m} \\
0 & -m & -\beta \frac{(a+m)(b+m)(c+m)}{m} \\
\alpha^{-1} & \beta^{-1} & a+b+c+m
\end{array}\right) .
$$

Hence

$$
\text { T. } x_{m} \cap \mathcal{F}=\left\{x_{m}\right\} \text {. }
$$

The characteristic polynomial of $x_{m}(a, b, c)$ is $(X-a)(X-b)(X-c)$. Moreover, $\operatorname{dim} B \cdot x_{m}(a, b, c)=5$, so that $\operatorname{dim} \mathcal{O}_{x_{m}(a, b, c)}=6$. We have shown that $x_{m}(a, b, c)$ is regular for every choice of $a, b, c \in k^{*}$. Now let $g$ be a regular element of GL(3). Since $\mathcal{O}_{g}$ is determined by the characteristic polynomial of $g$, there exist $a, b, c \in k^{*}$ such that $x_{m}(a, b, c) \in \mathcal{O}_{g}$ for every $m \in k^{*}$. We take $x_{m}=x_{m}(a, b, c)$ for $m \in k^{*}$. The set $\mathcal{F}=\left\{x_{m} \mid m \in k^{*}\right\}$ is the required set. 
Proposition 3.15. Let $s$ be a semisimple element of $\operatorname{SL}(n+1)$ with at most 2 eigenvalues, any characteristic, and $\mathcal{O}$ its conjugacy class. Then $\mathcal{O} \cap B w_{\mathcal{O}} B \cap B^{-}$ is nonempty, and $\mathcal{O}$ is spherical.

Proof. We may assume $s=\operatorname{diag}\left(a I_{k}, b I_{n+1-k}\right)$ with $a \neq b, 1 \leq k \leq\left[\frac{1}{2}(n+1)\right]$. Let $g=n_{\beta_{1}} \cdots n_{\beta_{k}} x_{\beta_{1}}(1) \cdots x_{\beta_{k}}(1)$. Then, by Lemma 3.7, $g_{s} g^{-1} \in \mathcal{O} \cap B w B \cap B^{-}$with $w=w_{\beta_{1}} \cdots w_{\beta_{k}}$. As $\operatorname{dim} \mathcal{O}=\ell(w)+\operatorname{rk}(1-w)$, we conclude by Proposition 3.6.

Theorem 3.16. Let $g$ be an element of $\mathrm{SL}(n+1)$, any characteristic, $g=s u$ its Jordan decomposition and $\mathcal{O}$ its conjugacy class. Then $\mathcal{O}$ is spherical if and only if one of the following holds:

(a) $u=1$ and $s$ has at most 2 eigenvalues.

(b) $u \neq 1, s \in Z(G)$ and $u$ has Jordan blocks of sizes at most 2 .

Proof. Assume that $\mathcal{O}$ is spherical. Suppose that neither (a) nor (b) hold. Since by [Knop 1995, Theorem 2.2] every conjugacy class contained in the closure of $\mathcal{O}$ is spherical, without loss of generality we may assume

$$
g=\operatorname{diag}(R, S) \quad \text { for } R \in \mathrm{GL}(3), S \in \mathrm{GL}(n-2), S \text { diagonal }
$$

with

$$
R=\left(\begin{array}{lll}
a & 1 & 0 \\
0 & a & 1 \\
0 & 0 & a
\end{array}\right) \text { or }\left(\begin{array}{lll}
a & 1 & 0 \\
0 & a & 0 \\
0 & 0 & b
\end{array}\right) \text { or }\left(\begin{array}{lll}
a & 0 & 0 \\
0 & b & 0 \\
0 & 0 & c
\end{array}\right) \text {, }
$$

$a, b$ and $c$ pairwise distinct. Hence $R$ is regular in GL(3). Consider the elements

$$
g_{m}=\operatorname{diag}\left(x_{m}, S\right)
$$

for $m \in k^{*}$, where $x_{m}$ is as defined in Lemma 3.14. We apply Lemma 3.10 with $J=\{1,2\}$ and $\mathcal{F}=\left\{g_{m} \mid m \in k^{*}\right\} \subset L_{J}$. The $g_{m}$ are all $G$-conjugate to $g$, and pairwise not $B_{J}$-conjugate. By Lemma 3.10 the family $\left(B . g_{m}\right)_{m \in k^{*}}$ is an infinite family of (distinct) $B$-orbits, a contradiction. Hence either (a) or (b) holds.

The remaining assertions follow by Proposition 3.15, and from the classification

\begin{tabular}{|c|c|c|c|c|}
\hline $\mathcal{O}$ & $J$ & $w_{\mathcal{O}}$ & $C(g)$ & $\operatorname{dim} \mathcal{O}$ \\
\hline $\begin{array}{c}\operatorname{diag}\left(a I_{k}, b I_{n+1-k}\right) \\
k=1, \ldots,\left[\frac{1}{2}(n+1)\right] \\
a \neq b\end{array}$ & $J_{k}$ & $s_{\beta_{1}} \cdots s_{\beta_{k}}$ & $T_{1} A_{k-1} A_{n-k}$ & $2 k(n+1-k)$ \\
\hline
\end{tabular}
of unipotent classes in zero or odd characteristic ([Carnovale 2010, Theorem 3.2] and in characteristic 2, [Costantini 2012, Table 1]).

Table 1. Spherical semisimple classes in $A_{n}$, where $w_{\mathcal{O}}=w_{0} w_{J}$ and $J_{k}=\{k+1, \ldots, n-k\}$ for $k=1, \ldots,\left[\frac{1}{2}(n+1)\right]-1, J_{\left[\frac{1}{2}(n+1)\right]}=\varnothing$. 
Type $\boldsymbol{C}_{\boldsymbol{n}}\left(\right.$ and $\left.\boldsymbol{B}_{\boldsymbol{n}}\right), \boldsymbol{p}=\mathbf{2}, \boldsymbol{n} \geq \mathbf{2}$. We put $\beta_{i}=2 e_{i}$ for each $i=1, \ldots, n$ and $\gamma_{\ell}=e_{2 \ell-1}+e_{2 \ell}$ for $\ell=1, \ldots,\left[\frac{1}{2} n\right]$.

We describe $G$ as the subgroup of GL(2n) of matrices preserving the bilinear form associated with the matrix $\left(\begin{array}{cc}0 & I_{n} \\ -I_{n} & 0\end{array}\right)$ with respect to the canonical basis of $k^{2 n}$. We observe that in characteristic 2 the groups of type $B_{n}$ and $C_{n}$ are isomorphic as abstract groups, hence we deal only with type $C_{n}$.

Proposition 3.17. Let $x$ be an element of $\operatorname{Sp}(2 n)$, any characteristic, $n \geq 2$, and $\mathcal{O}$ its conjugacy class. If either

(a) $x=a_{\lambda}=\operatorname{diag}\left(\lambda I_{n}, \lambda^{-1} I_{n}\right)$

for $\lambda \neq \pm 1$, or

(b) $x=c_{\lambda}=\operatorname{diag}\left(\lambda, I_{n-1}, \lambda^{-1}, I_{n-1}\right) \quad$ for $\lambda \neq \pm 1$,

then $\mathcal{O} \cap B w_{\mathcal{O}} B \cap B^{-}$is nonempty, $\operatorname{dim} \mathcal{O}=\ell\left(w_{\mathcal{O}}\right)+\operatorname{rk}\left(1-w_{\mathcal{O}}\right)$ and $\mathcal{O}$ is spherical. Proof. The proof uses the same method as the proof of Proposition 3.15, so we omit it.

Proposition 3.18. Let $G=\mathrm{Sp}(2 n), p=2, n \geq 2$. The spherical semisimple classes are represented by

(a) $a_{\lambda}=\operatorname{diag}\left(\lambda I_{n}, \lambda^{-1} I_{n}\right)$

for $\lambda \neq 1$,

(b) $c_{\lambda}=\operatorname{diag}\left(\lambda, I_{n-1}, \lambda^{-1}, I_{n-1}\right) \quad$ for $\lambda \neq 1$.

Proof. Let $x$ be a semisimple element of $G$, and assume $\mathcal{O}=\mathcal{O}_{x}$ is spherical. Without loss of generality $C(x)=L_{J}$, a pseudo-Levi subgroup of $G$. There exists a semisimple element $\tilde{x}$ in $G_{\mathbb{C}}$ such that $C(\tilde{x})$ is $L_{J}$ in $G_{\mathbb{C}}$. By Lemma 3.11, it follows that $\mathcal{O}_{\tilde{x}}$ is a spherical semisimple conjugacy class in $G_{\mathbb{C}}$, and therefore, from the classification of semisimple spherical conjugacy classes in zero (or odd) characteristic [Cantarini et al. 2005, Table 1; Carnovale 2010, Theorem 3.3], it follows that $L_{J}$ is of type $C_{\ell} C_{n-\ell}$ for $\ell=1, \ldots,\left[\frac{1}{2} n\right], T_{1} C_{n-1}$ or $T_{1} \widetilde{A}_{n-1}$. But $Z\left(C_{\ell} C_{n-\ell}\right)=1$, so that we are left with

$$
\begin{aligned}
& a_{\lambda}=\operatorname{diag}\left(\lambda I_{n}, \lambda^{-1} I_{n}\right) \longleftrightarrow T_{1} \widetilde{A}_{n-1}, \\
& c_{\lambda}=\operatorname{diag}\left(\lambda, I_{n-1}, \lambda^{-1}, I_{n-1}\right) \longleftrightarrow T_{1} C_{n-1},
\end{aligned}
$$

for $\lambda \neq 1$. We conclude by Proposition 3.17.

We now deal with mixed conjugacy classes.

Lemma 3.19. Let $H=\mathrm{Sp}(4)$, any characteristic, and

$$
g=\left(\begin{array}{cccc}
a & 0 & 0 & 0 \\
0 & 1 & 0 & 1 \\
0 & 0 & \frac{1}{a} & 0 \\
0 & 0 & 0 & 1
\end{array}\right),
$$


a mixed regular element of $H$ (so $a \neq \pm 1)$. Then there is a subset $\mathcal{F}=\left\{x_{m} \mid m \in k^{*}\right\}$ of $\mathcal{O}_{g}$ such that $\left(B(H) . x_{m}\right)_{m \in k^{*}}$ consists of pairwise distinct $B(H)$-orbits.

Proof. For $m \in k^{*}$, we put

$$
\begin{aligned}
x_{m} & =\left(\begin{array}{cccc}
0 & 0 & -\frac{1}{m} & 0 \\
0 & 0 & -1 & 1 \\
m & m & \frac{a^{2}+m+1}{a} & \frac{m(-2 a+m+1)}{a} \\
0 & -1 & -\frac{1}{a} & 2-\frac{m}{a}
\end{array}\right) \\
& =\left(\begin{array}{rrrr}
0 & 0 & -1 & 0 \\
0 & 0 & 0 & -1 \\
1 & 0 & 0 & 0 \\
0 & 1 & 0 & 0
\end{array}\right)\left(\begin{array}{rrrr}
m & 0 & 0 & 0 \\
0 & -1 & 0 & 0 \\
0 & 0 & \frac{1}{m} & 0 \\
0 & 0 & 0 & -1
\end{array}\right)\left(\begin{array}{cccc}
1 & 1 & \frac{a^{2}+m+1}{a m} & \frac{-2 a+m+1}{a} \\
0 & 1 & \frac{1}{a} & \frac{m}{a}-2 \\
0 & 0 & 1 & 0 \\
0 & 0 & -1 & 1
\end{array}\right) \in w_{0} B .
\end{aligned}
$$

The characteristic polynomial of $x_{m}$ is $(X-1)^{2}(X-a)(X-1 / a)$, and the 1eigenspace has dimension 1. Hence $x_{m}$ is $H$-conjugate to $g$. Suppose $x_{m}, x_{m^{\prime}}$ are $B$-conjugate. Then $x_{m}, x_{m^{\prime}}$ are $T$-conjugate and, from a direct calculation, it follows that T. $x_{m} \cap \mathcal{F}=\left\{x_{m}\right\}$, hence $m=m^{\prime}$.

Proposition 3.20. Let $\mathcal{O}$ be the conjugacy class of a mixed element $g$ of $\operatorname{Sp}(2 n)$, $p=2$. Then $\mathcal{O}$ is not spherical.

Proof. Let $g=s u$, the Jordan decomposition. Assume, for a contradiction, that $\mathcal{O}$ is spherical. Then both $\mathcal{O}_{s}$ and $\mathcal{O}_{u}$ are spherical. By Proposition 3.18, $H=C(s)$ is of type $T_{1} C_{n-1}$ or $T_{1} \widetilde{A}_{n-1}$. However $\operatorname{dim} T_{1} \widetilde{A}_{n-1}=n^{2}$, and therefore $C_{T_{1} \widetilde{A}_{n-1}}(u)$ is not spherical in $G$. We are left with $H$ of type $T_{1} C_{n-1}$, and we may assume $s=c_{a}=h_{\beta_{1}}(a)$ for a certain $a \neq 1$.

Since every conjugacy class contained in the closure of $\mathcal{O}$ is spherical, it is enough to deal with the minimal nontrivial spherical unipotent classes in $T_{1} C_{n-1}$. From the classification of spherical unipotent classes in characteristic 2 [Costantini 2012, Tables 1 and 2], we may assume

$$
\begin{aligned}
g & =h_{\beta_{1}}(a) x_{\alpha_{2}}(1) \quad \text { if } n=2, \\
g=h_{\beta_{n-1}}(a) x_{\alpha_{n}}(1) \quad \text { or } \quad g & =h_{\beta_{1}}(a) x_{\alpha_{2}}(1) \quad \text { if } n \geq 3,
\end{aligned}
$$

since $h_{\beta_{n-1}}(a)=\operatorname{diag}\left(I_{n-2}, a, 1, I_{n-2}, a^{-1}, 1\right)$ is conjugate to $h_{\beta_{1}}(a)$.

Suppose $g=h_{\beta_{n-1}}(a) x_{\alpha_{n}}(1), n \geq 2$. We apply Lemma 3.10 with $J=\{n-1, n\}$. By considering the corresponding embedding of $C_{2}$ into $C_{n}$, we may assume that the family $\mathcal{F}=\left\{x_{m} \mid m \in k^{*}\right\}$, introduced in Lemma 3.19, is a subset of $L_{J}$. The 
$x_{m}$ are all $G$-conjugate to $g$, and pairwise not $B_{J}$-conjugate. By Lemma 3.10, the family $\left(B . x_{m}\right)_{m \in k^{*}}$ is an infinite family of (distinct) $B$-orbits, a contradiction. Hence the class of $g=h_{\beta_{n-1}}(a) x_{\alpha_{n}}(1)$ is not spherical.

Suppose $g=h_{\beta_{1}}(a) x_{\alpha_{2}}(1), n \geq 3$. Then

$$
g=\left(\begin{array}{cccc}
A & 0 & 0 & 0 \\
0 & I_{n-3} & 0 & 0 \\
0 & 0 & { }^{t} A^{-1} & 0 \\
0 & 0 & 0 & I_{n-3}
\end{array}\right), \quad A=\left(\begin{array}{lll}
a & 0 & 0 \\
0 & 1 & 1 \\
0 & 0 & 1
\end{array}\right) .
$$

Let $\left(x_{m}\right)_{m \in k^{*}}$ be the family introduced in Lemma 3.14, such that $x_{m}$ is GL(3)conjugate to $A$ for every $m \in k^{*}$. We put

$$
g_{m}=\left(\begin{array}{cccc}
x_{m} & 0 & 0 & 0 \\
0 & I_{n-3} & 0 & 0 \\
0 & 0 & { }^{t} x_{m}^{-1} & 0 \\
0 & 0 & 0 & I_{n-3}
\end{array}\right) .
$$

The $g_{m}$ are all $\mathrm{Sp}(2 n)$-conjugate to $g$. By Lemma 3.10 with $J=\{1,2\}$, the family $\left(B . g_{m}\right)_{m \in k^{*}}$ is an infinite family of (distinct) $B$-orbits, a contradiction. Hence the class of $g=h_{\beta_{1}}(a) x_{\alpha_{2}}(1)$ is not spherical.

Theorem 3.21. Let $G=\operatorname{Sp}(2 n), p=2, n \geq 2$. The spherical classes are either semisimple or unipotent. The semisimple classes are represented in Table 2 and the unipotent classes are represented in Table 2 of [Costantini 2012].

\begin{tabular}{|c|cccc|}
\hline $\mathcal{O}$ & $J$ & $w_{\mathcal{O}}$ & $C(g)$ & $\operatorname{dim} \mathcal{O}$ \\
\hline $\begin{array}{c}c_{\lambda}=\operatorname{diag}\left(\lambda, I_{n-1}, \lambda^{-1}, I_{n-1}\right) \\
\lambda \neq 1\end{array}$ & $J_{2}$ & $s_{\beta_{1}} s_{\beta_{2}}$ & $T_{1} C_{n-1}$ & $4 n-2$ \\
\hline $\begin{array}{c}a_{\lambda}=\operatorname{diag}\left(\lambda I_{n}, \lambda^{-1} I_{n}\right) \\
\lambda \neq 1\end{array}$ & $\varnothing$ & $w_{0}=s_{\beta_{1}} \cdots s_{\beta_{n}}$ & $T_{1} \widetilde{A}_{n-1}$ & $n^{2}+n$ \\
\hline
\end{tabular}

Table 2. Spherical semisimple classes in $C_{n}, n \geq 2, p=2$. Here $w_{\mathcal{O}}=w_{0} w_{J}, J_{2}=\varnothing$ if $n=2$ and $J_{2}=\{3, \ldots, n\}$ if $n \geq 3$.

Type $D_{n}, \boldsymbol{p}=2, \boldsymbol{n} \geq 4$. Let $r=\left[\frac{1}{2} n\right]$. We put $\beta_{\ell}=e_{2 \ell-1}+e_{2 \ell}$ and $\delta_{\ell}=e_{2 \ell-1}-e_{2 \ell}$ for $\ell=1, \ldots, r$. Also, we set $J_{1}=\{3, \ldots, n\}, K_{r}=\{1,3, \ldots, 2 r-1\}$ and, if $n$ is even, $K_{r}^{\prime}=\{1,3, \ldots, n-3, n\}$.

In this section we deal with groups $G$ of type $D_{n}$. We recall that we are assuming $G$ simply connected. Since $p=2$, the covering map $\pi: G \rightarrow \operatorname{SO}(2 n)$ is an isomorphism of abstract groups. We describe $\mathrm{SO}(2 n)$ as the connected component 
of the subgroup of $\operatorname{Sp}(2 n)$ of matrices preserving the quadratic form associated with $\left(\begin{array}{cc}0 & I_{n} \\ I_{n} & 0\end{array}\right)$ with respect to the canonical basis of $k^{2 n}$ [Carter 1989, §1.6].

Proposition 3.22. Let $x$ be an element of $G=D_{n}$, any characteristic, $n \geq 4$, and $\mathcal{O}$ its conjugacy class. If one of

(a) $x=c_{\lambda}=h_{\beta_{1}}(\lambda) h_{\delta_{1}}(\lambda)$

for $\lambda \neq \pm 1$,

(b) $x=a_{\lambda}=h_{\beta_{1}}(\lambda) \cdots h_{\beta_{r}}(\lambda)$

for $\lambda \neq \pm 1$, or

(c) $x=a_{\lambda}^{\prime}=h_{\beta_{1}}(\lambda) \cdots h_{\beta_{r-1}}(\lambda) h_{\alpha_{n-1}}(\lambda)$

for $\lambda \neq \pm 1, n$ even,

holds, then $\mathcal{O} \cap B w_{\mathcal{O}} B \cap B^{-}$is nonempty, $\operatorname{dim} \mathcal{O}=\ell\left(w_{\mathcal{O}}\right)+\operatorname{rk}\left(1-w_{\mathcal{O}}\right)$ and $\mathcal{O}$ is spherical.

Proof. Assume $x=c_{\lambda}$ with $\lambda \neq \pm 1$. Let $g=n_{\beta_{1}} n_{\delta_{1}} x_{\beta_{1}}(1) x_{\delta_{1}}$ (1). Then we have $g x g^{-1} \in \mathcal{O} \cap B w B \cap B^{-}$, with $w=s_{\beta_{1}} s_{\delta_{1}}$ and $\operatorname{dim} \mathcal{O}=\ell(w)+\operatorname{rk}(1-w)$.

Similarly, assume $x=a_{\lambda}$ with $\lambda \neq \pm 1$. Let $g=n_{\beta_{1}} \cdots n_{\beta_{r}} x_{\beta_{1}}(1) \cdots x_{\beta_{r}}(1)$. Then $g x g^{-1} \in \mathcal{O} \cap B w B \cap B^{-}$, with $w=s_{\beta_{1}} \cdots s_{\beta_{r}}$ and $\operatorname{dim} \mathcal{O}=\ell(w)+\operatorname{rk}(1-w)$.

The case (c) follows from (b) by using the graph automorphism of $G$ exchanging $n-1$ and $n$. We conclude by Proposition 3.6.

Proposition 3.23. Let $G=D_{n}, p=2, n \geq 4$. The spherical semisimple classes are represented by

(a) $x=c_{\lambda}=h_{\beta_{1}}(\lambda) h_{\delta_{1}}(\lambda)$

for $\lambda \neq 1$,

(b) $x=a_{\lambda}=h_{\beta_{1}}(\lambda) \cdots h_{\beta_{m}}(\lambda)$

for $\lambda \neq 1$,

(c) $x=a_{\lambda}^{\prime}=h_{\beta_{1}}(\lambda) \cdots h_{\beta_{m-1}}(\lambda) h_{\alpha_{n-1}}(\lambda)$

( $\lambda$ ) for $\lambda \neq 1, n$ even.

Proof. The proof uses the same method as the proof of Proposition 3.18, so we omit it.

We now deal with mixed conjugacy classes.

Proposition 3.24. Let $\mathcal{O}$ be the conjugacy class of a mixed element $g$ in $D_{n}, p=2$. Then $\mathcal{O}$ is not spherical.

Proof. We work with $\mathrm{SO}(2 n)$ via $\pi$. Let $g=s u$, the Jordan decomposition. Assume that $\mathcal{O}$ is spherical. Then both $\mathcal{O}_{s}$ and $\mathcal{O}_{u}$ are spherical, and we may assume, up to conjugation and graph automorphism, that for a certain $a \neq 1$,

$$
s=\operatorname{diag}\left(a I_{n-1}, a^{-1}, a^{-1} I_{n-1}, a\right) \quad \text { or } \quad s=\operatorname{diag}\left(I_{n-3}, a, I_{2}, I_{n-3}, a^{-1}, I_{2}\right) .
$$

Assume $s=\operatorname{diag}\left(a I_{n-1}, a^{-1}, a^{-1} I_{n-1}, a\right)$ for a certain $a \neq 1$. Without loss of generality we may assume $u=x_{\alpha_{n-2}}\left(a^{-1}\right)$, so that

$$
g=\left(\begin{array}{cccc}
a I_{n-3} & 0 & 0 & 0 \\
0 & A & 0 & 0 \\
0 & 0 & a^{-1} I_{n-3} & 0 \\
0 & 0 & 0 & { }^{t} A^{-1}
\end{array}\right), \quad A=\left(\begin{array}{ccc}
a & 1 & 0 \\
0 & a & 0 \\
0 & 0 & a^{-1}
\end{array}\right) .
$$


Let $\left(x_{m}\right)_{m \in k^{*}}$ be the family introduced in Lemma 3.14, such that $x_{m}$ is GL(3)conjugate to $A$ for every $m \in k^{*}$ and $\left(B(\mathrm{GL}(3)) \cdot x_{m}\right)_{m \in k^{*}}$ consists of pairwise distinct $B(\mathrm{GL}(3))$-orbits.

We put

$$
g_{m}=\left(\begin{array}{cccc}
a I_{n-3} & 0 & 0 & 0 \\
0 & x_{m} & 0 & 0 \\
0 & 0 & a^{-1} I_{n-3} & 0 \\
0 & 0 & 0 & { }^{t} x_{m}^{-1}
\end{array}\right) .
$$

The $g_{m}$ are all $\mathrm{SO}(2 n)$-conjugate to $g$. By Lemma 3.10 with $J=\{n-1, n-2\}$, the family $\left(B . g_{m}\right)_{m \in k^{*}}$ is an infinite family of (distinct) $B$-orbits, a contradiction. This settles the cases when $n$ is odd and $C(s)$ is of type $T_{1} A_{n-1}$, and when $n$ is even and $C(s)$ is of type $\left(T_{1} A_{n-1}\right)^{\prime}$. Upon application of the graph automorphism exchanging $n$ and $n-1$, this also settles the case when $n$ is even and $C(s)$ is of type $T_{1} A_{n-1}$.

Assume $s=\operatorname{diag}\left(a, I_{n-1}, a^{-1}, I_{n-1}\right)$ for a certain $a \neq 1$. Without loss of generality we may assume $u=x_{\alpha_{2}}(1)$, so that

$$
g=\left(\begin{array}{cccc}
A & 0 & 0 & 0 \\
0 & I_{n-3} & 0 & 0 \\
0 & 0 & { }^{t} A^{-1} & 0 \\
0 & 0 & 0 & I_{n-3}
\end{array}\right), \quad A=\left(\begin{array}{lll}
a & 0 & 0 \\
0 & 1 & 1 \\
0 & 0 & 1
\end{array}\right) .
$$

Let $\left(x_{m}\right)_{m \in k^{*}}$ be the family introduced in Lemma 3.14, such that $x_{m}$ is GL(3)conjugate to $A$ for every $m \in k^{*}$ and $\left(B(\mathrm{GL}(3)) \cdot x_{m}\right)_{m \in k^{*}}$ consists of pairwise distinct $B(\mathrm{GL}(3))$-orbits.

\begin{tabular}{|c|cccc|}
\hline $\mathcal{O}$ & $J$ & $w_{\mathcal{O}}$ & $C(g)$ & $\operatorname{dim} \mathcal{O}$ \\
\hline$c_{\lambda}=h_{\beta_{1}}(\lambda) h_{\delta_{1}}(\lambda)$ & & & & \\
$\lambda \neq 1$ & $J_{1}$ & $s_{\beta_{1}} s_{\delta_{1}}$ & $T_{1} D_{n-1}$ & $4(n-1)$ \\
$\operatorname{diag}\left(\lambda^{2}, I_{n-1}, \lambda^{-2}, I_{n-1}\right)$ & & & & \\
\hline $\begin{array}{c}a_{\lambda}=h_{\beta_{1}}(\lambda) \cdots h_{\beta_{r}}(\lambda) \\
\lambda \neq 1\end{array}$ & $K_{r}$ & $s_{\beta_{1}} \cdots s_{\beta_{r}}$ & $T_{1} A_{n-1}$ & $n^{2}-n$ \\
$\operatorname{diag}\left(\lambda I_{n}, \lambda^{-1} I_{n}\right)$ & & & & \\
\hline $\begin{array}{c}a_{\lambda}^{\prime}=h_{\beta_{1}}(\lambda) \cdots h_{\beta_{r-1}}(\lambda) h_{\alpha_{n-1}}(\lambda) \\
\lambda \neq 1 \\
\operatorname{diag}\left(\lambda I_{n-1}, \lambda^{-1}, \lambda^{-1} I_{n-1}, \lambda\right)\end{array}$ & $K_{r}^{\prime}$ & $s_{\beta_{1}} \cdots s_{\beta_{r-1}} s_{\alpha_{n-1}}$ & $\left(T_{1} A_{n-1}\right)^{\prime}$ & $n^{2}-n$ \\
\hline
\end{tabular}

Table 3. Spherical semisimple classes in $D_{n}, p=2, n \geq 4, n=2 r$, where $w_{\mathcal{O}}=w_{0} w_{J}$. 


\begin{tabular}{|c|cccc|}
\hline $\mathcal{O}$ & $J$ & $w_{\mathcal{O}}$ & $C(g)$ & $\operatorname{dim} \mathcal{O}$ \\
\hline$c_{\lambda}=h_{\beta_{1}}(\lambda) h_{\delta_{1}}(\lambda)$ & & & & \\
$\lambda \neq 1$ & $J_{1}$ & $s_{\beta_{1}} s_{\delta_{1}}$ & $T_{1} D_{n-1}$ & $4(n-1)$ \\
$\operatorname{diag}\left(\lambda^{2}, I_{n-1}, \lambda^{-2}, I_{n-1}\right)$ & & & & \\
\hline $\begin{array}{c}a_{\lambda}=h_{\beta_{1}}(\lambda) \cdots h_{\beta_{r}}(\lambda) \\
\lambda \neq 1\end{array}$ & & & & \\
$\operatorname{diag}\left(\lambda I_{n}, \lambda^{-1} I_{n}\right)$ & $K_{r}$ & $s_{\beta_{1}} \cdots s_{\beta_{r}}$ & $T_{1} A_{n-1}$ & $n^{2}-n$ \\
\hline
\end{tabular}

Table 4. Spherical semisimple classes in $D_{n}, p=2, n \geq 5$, $n=2 r+1$, where $w_{\mathcal{O}}=w_{0} w_{J}$.

Set

$$
g_{m}=\left(\begin{array}{cccc}
x_{m} & 0 & 0 & 0 \\
0 & I_{n-3} & 0 & 0 \\
0 & 0 & { }^{t} x_{m}^{-1} & 0 \\
0 & 0 & 0 & I_{n-3}
\end{array}\right) .
$$

The $g_{m}$ are all $\mathrm{SO}(2 n)$-conjugate to $g$. By Lemma 3.10 with $J=\{1,2\}$, the family $\left(B . g_{m}\right)_{m \in k^{*}}$ is an infinite family of (distinct) $B$-orbits, a contradiction. This settles the case when $C(s)$ is of type $T_{1} D_{n-1}$, and we are done.

Theorem 3.25. Let $G=D_{n}, p=2, n \geq 4$. The spherical classes are either semisimple or unipotent. The semisimple classes are represented in Tables 3 and 4, and the unipotent classes in Tables 3 and 4 of [Costantini 2012].

Type $\boldsymbol{E}_{6}$. We put

$$
\begin{array}{ll}
\beta_{1}=(1,2,2,3,2,1), & \beta_{2}=(1,0,1,1,1,1), \\
\beta_{3}=(0,0,1,1,1,0), & \beta_{4}=(0,0,0,1,0,0) .
\end{array}
$$

Proposition 3.26. Let $x$ be an element of $E_{6}$, any characteristic, and $\mathcal{O}$ its conjugacy class. If one of

(a) $x=h_{\alpha_{1}}(-1) h_{\alpha_{4}}(-1) h_{\alpha_{6}}(-1)$,

(b) $x=h(z)=h_{\alpha_{1}}\left(z^{4}\right) h_{\alpha_{2}}\left(z^{3}\right) h_{\alpha_{3}}\left(z^{5}\right) h_{\alpha_{4}}\left(z^{6}\right) h_{\alpha_{5}}\left(z^{4}\right) h_{\alpha_{6}}\left(z^{2}\right) \quad$ for $z^{3} \neq 1$,

holds, then $\mathcal{O} \cap B w_{\mathcal{O}} B \cap B^{-}$is nonempty, $\operatorname{dim} \mathcal{O}=\ell\left(w_{\mathcal{O}}\right)+\operatorname{rk}\left(1-w_{\mathcal{O}}\right)$, and $\mathcal{O}$ is spherical.

Proof. (a) If $p=2$, then $x=1$, and there is nothing to prove. So assume $p \neq 2$. In $G$ there are two classes of involutions: one has centralizer of type $A_{1} A_{5}$ and dimension 40 , the other has centralizer of type $D_{5} T_{1}$ and has dimension 32 . Let $y=n_{\beta_{1}} \cdots n_{\beta_{4}} \in w_{0} B, w=s_{\beta_{1}} \cdots s_{\beta_{4}}=w_{0}$. Then $y^{2}=h_{\beta_{1}}(-1) \cdots h_{\beta_{4}}(-1)=1$, and $\operatorname{dim} \mathcal{O}_{y} \geq 40$ by Proposition 3.6. Since $C(x)$ is of type $A_{1} A_{5}$, we conclude 
that $x \sim y$, so that $\mathcal{O} \cap B w_{0} B$ is nonempty, $\operatorname{dim} \mathcal{O}=\ell\left(w_{0}\right)+\operatorname{rk}\left(1-w_{0}\right)$ and $\mathcal{O}$ is spherical. It is a general fact that if $t$ is semisimple and $\mathcal{O}_{t} \cap B w B \neq \varnothing$, then $\mathcal{O}_{t} \cap B w B \cap B^{-} \neq \varnothing$ [Cantarini et al. 2005, Lemma 14].

(b) In this case $C(x)$ is of type $D_{5} T_{1}$ (note that $C(x)=C(h(-1))$ if $p \neq 2$ ). Let $g=n_{\beta_{1}} n_{\beta_{2}} x_{\beta_{1}}(1) x_{\beta_{2}}(1)$. Then $g x g^{-1} \in \mathcal{O} \cap B s_{\beta_{1}} s_{\beta_{2}} B \cap B^{-}$, with $w=s_{\beta_{1}} s_{\beta_{2}}$ and $\operatorname{dim} \mathcal{O}=\ell(w)+\operatorname{rk}(1-w)$. We conclude by Proposition 3.6.

Proposition 3.27. Let $G=E_{6}$. The spherical semisimple classes are represented by

$$
\begin{aligned}
& h(z)=h_{\alpha_{1}}\left(z^{4}\right) h_{\alpha_{2}}\left(z^{3}\right) h_{\alpha_{3}}\left(z^{5}\right) h_{\alpha_{4}}\left(z^{6}\right) h_{\alpha_{5}}\left(z^{4}\right) h_{\alpha_{6}}\left(z^{2}\right), z^{3} \neq 1, \quad \text { for } p=2 \text {, } \\
& \left.\begin{array}{l}
h_{\alpha_{1}}(-1) h_{\alpha_{4}}(-1) h_{\alpha_{6}}(-1), \\
h(z)=h_{\alpha_{1}}\left(z^{4}\right) h_{\alpha_{2}}\left(z^{3}\right) h_{\alpha_{3}}\left(z^{5}\right) h_{\alpha_{4}}\left(z^{6}\right) h_{\alpha_{5}}\left(z^{4}\right) h_{\alpha_{6}}\left(z^{2}\right), z \neq 1,
\end{array}\right\} \text { for } p=3 .
\end{aligned}
$$

Proof. Let $x$ be a semisimple element of $G$, and assume $\mathcal{O}=\mathcal{O}_{x}$ is spherical. Without loss of generality $C(x)=L_{J}$, a pseudo-Levi subgroup of $G$. There exists a semisimple element $\tilde{x}$ in $G_{\mathbb{C}}$ such that $C(\tilde{x})$ is $L_{J}$ in $G_{\mathbb{C}}$. By Lemma 3.11, it follows that $\mathcal{O}_{\tilde{x}}$ is a spherical semisimple conjugacy class in $G_{\mathbb{C}}$, and therefore, from the classification of semisimple spherical conjugacy classes in zero (or good odd) characteristic [Cantarini et al. 2005, Table 2; Carnovale 2010, Theorem 3.6], it follows that $L_{J}$ is of type $A_{1} A_{5}$ or $D_{5} T_{1}$.

Let $p=2$. Then $Z\left(A_{1} A_{5}\right)=Z(G)$ (of order 3 ), so that we are left with $h(z)$, for $z^{3} \neq 1$.

Let $p=3$. Then $Z(G)=1$, and we conclude by Proposition 3.26.

We have established the information in Tables 5 and 6 , where $w_{\mathcal{O}}=w_{0} w_{J}$.

Proposition 3.28. Let $\mathcal{O}$ be the conjugacy class of a mixed element $g$ in $E_{6}, p=2$ or 3. Then $\mathcal{O}$ is not spherical.

Proof. Let $g=s u$, the Jordan decomposition. Assume that $\mathcal{O}$ is spherical. Then both $\mathcal{O}_{s}$ and $\mathcal{O}_{u}$ are spherical, and therefore $C(s)$ is of type $A_{1} A_{5}$ or $D_{5} T_{1}$. A dimensional argument rules out all the possibilities except the case that $C(s)$ is of type $A_{1} A_{5}$ and $u$ is a nonidentical unipotent element in the component $A_{1}$ of $C(s)$ (hence $p=3$ ). Therefore, without loss of generality we may assume $g=h_{\alpha_{1}}(-1) x_{\alpha_{1}}(1)$, which is a regular element of the standard Levi subgroup $L_{J}$, for $J=\{1,2\}$. By Lemma 3.14, there is an infinite family $\mathcal{F}=\left\{g_{m} \mid m \in k^{*}\right\} \subset L_{J}$ such that the $g_{m}$

\begin{tabular}{|c|cccc|}
\hline $\mathcal{O}$ & $J$ & $w_{\mathcal{O}}$ & $C(g)$ & $\operatorname{dim} \mathcal{O}$ \\
\hline $\begin{array}{c}h(z)=h_{\alpha_{3}}\left(z^{5}\right) h_{\alpha_{4}}\left(z^{6}\right) h_{\alpha_{5}}\left(z^{4}\right) h_{\alpha_{6}}\left(z^{2}\right) \\
z^{3} \neq 1\end{array}$ & $\{3,4,5\}$ & $s_{\beta_{1}} s_{\beta_{2}}$ & $D_{5} T_{1}$ & 32 \\
\hline
\end{tabular}

Table 5. Spherical semisimple classes in $E_{6}, p=2$. 


\begin{tabular}{|c|cccc|}
\hline $\mathcal{O}$ & $J$ & $w_{\mathcal{O}}$ & $C(g)$ & $\operatorname{dim} \mathcal{O}$ \\
\hline$h(z)=h_{\alpha_{1}}\left(z^{4}\right) h_{\alpha_{2}}\left(z^{3}\right) h_{\alpha_{3}}\left(z^{5}\right)$ & & & & \\
$\cdot h_{\alpha_{4}}\left(z^{6}\right) h_{\alpha_{5}}\left(z^{4}\right) h_{\alpha_{6}}\left(z^{2}\right)$ & $\{3,4,5\}$ & $s_{\beta_{1}} s_{\beta_{2}}$ & $D_{5} T_{1}$ & 32 \\
$z \neq 1$ & & & & \\
\hline$h_{\alpha_{1}}(-1) h_{\alpha_{4}}(-1) h_{\alpha_{6}}(-1) \sim h_{\alpha_{1}}(-1)$ & $\varnothing$ & $w_{0}$ & $A_{1} A_{5}$ & 40 \\
\hline
\end{tabular}

Table 6. Spherical semisimple classes in $E_{6}, p=3$.

are all $L_{J}$-conjugate (hence $G$-conjugate) to $g$, and pairwise not $B_{J}$-conjugate. By Lemma 3.10 the family $\left(B . g_{m}\right)_{m \in k^{*}}$ is an infinite family of (distinct) $B$-orbits, a contradiction. Hence $\mathcal{O}$ is not spherical.

Theorem 3.29. Let $G=E_{6}, p=2$ or 3 . The spherical classes are either semisimple or unipotent, up to a central element if $p=2$. The semisimple classes are represented in Tables 5 and 6, and the unipotent classes are represented in Tables 6 and 7 of [Costantini 2012].

Type $\boldsymbol{E}_{7}$. Here $Z(G)=\langle\tau\rangle$, where $\tau=h_{\alpha_{2}}(-1) h_{\alpha_{5}}(-1) h_{\alpha_{7}}(-1)$. We put

$$
\begin{gathered}
\beta_{1}=(2,2,3,4,3,2,1), \quad \beta_{2}=(0,1,1,2,2,2,1), \quad \beta_{3}=(0,1,1,2,1,0,0), \\
\beta_{4}=\alpha_{7}, \quad \beta_{5}=\alpha_{5}, \quad \beta_{6}=\alpha_{3}, \quad \beta_{7}=\alpha_{2} .
\end{gathered}
$$

Proposition 3.30. Let $x$ be an element of $E_{7}$, any characteristic, and $\mathcal{O}$ its conjugacy class. If one of

(a) $x=h_{\alpha_{2}}(-\zeta) h_{\alpha_{5}}(\zeta) h_{\alpha_{6}}(-1) h_{\alpha_{7}}(-\zeta)$ for $\zeta^{2}=-1, p \neq 2$,

(b) $x=h_{\alpha_{1}}(-1)$ for $p \neq 2$,

(c) $x=h(z)=h_{\alpha_{1}}\left(z^{2}\right) h_{\alpha_{2}}\left(z^{3}\right) h_{\alpha_{3}}\left(z^{4}\right) h_{\alpha_{4}}\left(z^{6}\right) h_{\alpha_{5}}\left(z^{5}\right) h_{\alpha_{6}}\left(z^{4}\right) h_{\alpha_{7}}\left(z^{3}\right) \quad$ for $z \neq \pm 1$, holds, then $\mathcal{O} \cap B w_{\mathcal{O}} B \cap B^{-}$is nonempty, $\operatorname{dim} \mathcal{O}=\ell\left(w_{\mathcal{O}}\right)+\operatorname{rk}\left(1-w_{\mathcal{O}}\right)$, and $\mathcal{O}$ is spherical.

Proof. (a) Let $Y$ be the set of elements $y$ of order 4 of $T$ such that $y^{2}=\tau$. Then $Y$ is the disjoint union of $2 W$-classes $Y_{1}$ and $Y_{2}: C(y)$ is of type $A_{7}$ if $y \in Y_{1}$, and of type $E_{6} T_{1}$ if $y \in Y_{2}$. A representative for $Y_{1}$ is $h_{\alpha_{2}}(-\zeta) h_{\alpha_{5}}(\zeta) h_{\alpha_{6}}(-1) h_{\alpha_{7}}(-\zeta)$ where $\zeta$ is a square root of -1 .

Let $y=n_{\beta_{1}} \cdots n_{\beta_{7}} \in w_{0} B, w=s_{\beta_{1}} \cdots s_{\beta_{7}}=w_{0}$. Then $y^{2}=h_{\beta_{1}}(-1) \cdots h_{\beta_{7}}(-1)=\tau$, and $\operatorname{dim} \mathcal{O}_{y} \geq \operatorname{dim} B$ by Proposition 3.6. Since $C(x)$ is of type $A_{7}$, we conclude that $x \sim y$, so that $\mathcal{O} \cap B w_{0} B$ is nonempty, $\operatorname{dim} \mathcal{O}=\ell\left(w_{0}\right)+\operatorname{rk}\left(1-w_{0}\right)$ and $\mathcal{O}$ is spherical. As above, $\mathcal{O} \cap B w B \cap B^{-} \neq \varnothing$.

(b) The group $G$ has 2 classes of noncentral involutions: $\mathcal{O}_{h_{\beta_{1}}(-1)}$ and $\mathcal{O}_{h_{\beta_{1}}(-1) \tau}$. In fact there are 127 involutions in $T$, and $\tau$ is central. The remaining 126 fall in 2 
classes: $\left\{h_{\alpha}(-1) \mid \alpha \in \Phi^{+}\right\}$and $\left\{h_{\alpha}(-1) \tau \mid \alpha \in \Phi^{+}\right\}$. Let $y=n_{\beta_{1}} n_{\beta_{2}} n_{\beta_{3}} n_{\alpha_{3}} \in w B$, where $w=s_{\beta_{1}} s_{\beta_{2}} s_{\beta_{3}} s_{\alpha_{3}}$. Then $y^{2}=h_{\beta_{1}}(-1) h_{\beta_{2}}(-1) h_{\beta_{3}}(-1) h_{\alpha_{3}}(-1)=1$, so that $y$ is a (noncentral) involution. We conclude that $x \sim y$ or $x \sim y \tau$, so that (in either case) $\mathcal{O} \cap B w B$ is nonempty, $\operatorname{dim} \mathcal{O}=\ell(w)+\operatorname{rk}(1-w)$ and $\mathcal{O}$ is spherical. As above, $\mathcal{O} \cap B w B \cap B^{-} \neq \varnothing$. (In fact we have $n_{\alpha} \sim h_{\alpha}(\zeta)$ already in $\left\langle X_{\alpha}, X_{-\alpha}\right\rangle$ for every root $\alpha$, hence $n_{\beta_{1}} n_{\beta_{2}} n_{\beta_{3}} n_{\alpha_{3}} \sim h_{\beta_{1}}(\zeta) h_{\beta_{2}}(\zeta) h_{\beta_{3}}(\zeta) h_{\alpha_{3}}(\zeta)=h_{\gamma}(-1)$, where $\gamma=\beta_{1}-\alpha_{1}$. Therefore $x \sim y$.)

(c) (any characteristic) We have $C(x)$ of type $E_{6} T_{1}$. Let

$$
g=n_{\beta_{1}} n_{\beta_{2}} n_{\alpha_{7}} x_{\beta_{1}}(1) x_{\beta_{2}}(1) x_{\alpha_{7}}(1) .
$$

Then $\operatorname{gxg}^{-1} \in \mathcal{O} \cap B w B \cap B^{-}$, with $w=s_{\beta_{1}} s_{\beta_{2}} s_{\alpha_{7}}$, and $\operatorname{dim} \mathcal{O}=\ell(w)+\operatorname{rk}(1-w)$. We conclude by Proposition 3.6.

Proposition 3.31. Let $G=E_{7}$. The spherical semisimple classes are represented by

$$
\left.\begin{array}{l}
h(z)=h_{\alpha_{1}}\left(z^{2}\right) h_{\alpha_{2}}\left(z^{3}\right) h_{\alpha_{3}}\left(z^{4}\right) h_{\alpha_{4}}\left(z^{6}\right) h_{\alpha_{5}}\left(z^{5}\right) h_{\alpha_{6}}\left(z^{4}\right) h_{\alpha_{7}}\left(z^{3}\right), z \neq 1, \quad \text { for } p=2, \\
h_{\alpha_{2}}(-\zeta) h_{\alpha_{5}}(\zeta) h_{\alpha_{6}}(-1) h_{\alpha_{7}}(-\zeta), \zeta^{2}=-1, \\
h_{\alpha_{1}}(-1), h_{\alpha_{1}}(-1) \tau, \\
h(z)=h_{\alpha_{1}}\left(z^{2}\right) h_{\alpha_{2}}\left(z^{3}\right) h_{\alpha_{3}}\left(z^{4}\right) h_{\alpha_{4}}\left(z^{6}\right) h_{\alpha_{5}}\left(z^{5}\right) h_{\alpha_{6}}\left(z^{4}\right) h_{\alpha_{7}}\left(z^{3}\right), z \neq \pm 1,
\end{array}\right\} \text { for } p=3 .
$$

Proof. Let $x$ be a semisimple element of $G$, and assume $\mathcal{O}=\mathcal{O}_{x}$ is spherical. Without loss of generality $C(x)=L_{J}$, a pseudo-Levi subgroup of $G$. There exists a semisimple element $\tilde{x}$ in $G_{\mathbb{C}}$ such that $C(\tilde{x})$ is $L_{J}$ in $G_{\mathbb{C}}$. By Lemma 3.11, it follows that $\mathcal{O}_{\tilde{x}}$ is a spherical semisimple conjugacy class in $G_{\mathbb{C}}$, and therefore, from the classification of semisimple spherical conjugacy classes in zero (or good odd) characteristic [Cantarini et al. 2005, Table 2; Carnovale 2010, Theorem 3.7], it follows that $L_{J}$ is of type $E_{6} T_{1}, D_{6} A_{1}$ or $A_{7}$.

Let $p=2$. Then $Z(G)=Z\left(D_{6} A_{1}\right)=Z\left(A_{7}\right)=1$, so that we are left with $h(z)$, for $z \neq 1$.

For $p=3$, we conclude by Proposition 3.30.

We have established the information in Tables 7 and 8 , where $w_{\mathcal{O}}=w_{0} w_{J}$.

\begin{tabular}{|c|cccc|}
\hline $\mathcal{O}$ & $J$ & $w_{\mathcal{O}}$ & $C(g)$ & $\operatorname{dim} \mathcal{O}$ \\
\hline$h(z)=h_{\alpha_{1}}\left(z^{2}\right) h_{\alpha_{2}}\left(z^{3}\right) h_{\alpha_{3}}\left(z^{4}\right)$ & & & & \\
$\cdot h_{\alpha_{4}}\left(z^{6}\right) h_{\alpha_{5}}\left(z^{5}\right) h_{\alpha_{6}}\left(z^{4}\right) h_{\alpha_{7}}\left(z^{3}\right)$ & $\{2,3,4,5\}$ & $s_{\beta_{1}} s_{\beta_{2}} s_{\alpha_{7}}$ & $E_{6} T_{1}$ & 54 \\
$z \neq 1$ & & & & \\
\hline
\end{tabular}

Table 7. Spherical semisimple classes in $E_{7}, p=2$. 


\begin{tabular}{|c|cccc|}
\hline $\mathcal{O}$ & $J$ & $w_{\mathcal{O}}$ & $C(g)$ & $\operatorname{dim} \mathcal{O}$ \\
\hline$h(z)=h_{\alpha_{1}}\left(z^{2}\right) h_{\alpha_{2}}\left(z^{3}\right) h_{\alpha_{3}}\left(z^{4}\right)$ & & & & \\
$\cdot h_{\alpha_{4}}\left(z^{6}\right) h_{\alpha_{5}}\left(z^{5}\right) h_{\alpha_{6}}\left(z^{4}\right) h_{\alpha_{7}}\left(z^{3}\right)$ & $\{2,3,4,5\}$ & $s_{\beta_{1}} s_{\beta_{2}} s_{\alpha_{7}}$ & $E_{6} T_{1}$ & 54 \\
$z \neq \pm 1$ & $\{2,5,7\}$ & $s_{\beta_{1}} s_{\beta_{2}} s_{\beta_{3}} s_{\alpha_{3}}$ & $D_{6} A_{1}$ & 64 \\
\hline$h_{\alpha_{1}}(-1), h_{\alpha_{1}}(-1) \tau$ & $\varnothing$ & $w_{0}$ & $A_{7}$ & 70 \\
\hline$h_{\alpha_{2}}(-\zeta) h_{\alpha_{5}}(\zeta) h_{\alpha_{6}}(-1) h_{\alpha_{7}}(-\zeta)$ & & & & \\
$\zeta^{2}=-1$ & & & & \\
\hline
\end{tabular}

Table 8. Spherical semisimple classes in $E_{7}, p=3$.

Proposition 3.32. Let $\mathcal{O}$ be the conjugacy class of a mixed element $g$ in $E_{7}, p=2$ or 3. Then $\mathcal{O}$ is not spherical.

Proof. Let $g=s u$, the Jordan decomposition. Assume that $\mathcal{O}$ is spherical. Then both $\mathcal{O}_{s}$ and $\mathcal{O}_{u}$ are spherical, and therefore $C(s)$ is of type $E_{6} T_{1}, D_{6} A_{1}$ or $A_{7}$. A dimensional argument rules out all the possibilities except the case that $C(s)$ is of type $D_{6} A_{1}$ and $u$ is a nonidentical unipotent element in the component $A_{1}$ of $C(s)$ (hence $p=3$ ). Therefore, without loss of generality we may assume $g=h_{\alpha_{7}}(-1) x_{\alpha_{7}}(1)$, which is a regular element of the standard Levi subgroup $L_{J}$, for $J=\{6,7\}$. By Lemma 3.14, there is an infinite family $\mathcal{F}=\left\{g_{m} \mid m \in k^{*}\right\} \subset L_{J}$ such that the $g_{m}$ are all $L_{J}$-conjugate (hence $G$-conjugate) to $g$, and pairwise not $B_{J}$-conjugate. By Lemma 3.10 the family $\left(B . g_{m}\right)_{m \in k^{*}}$ is an infinite family of (distinct) $B$-orbits, a contradiction. Hence $\mathcal{O}$ is not spherical.

Theorem 3.33. Let $G=E_{7}, p=2$ or 3 . The spherical classes are either semisimple or unipotent, up to a central element if $p=3$. The semisimple classes are represented in Tables 7 and 8, and the unipotent classes are represented in Tables 8 and 9 of [Costantini 2012].

Type $\boldsymbol{E}_{\mathbf{8}}$. We put

$$
\begin{aligned}
\beta_{1}=(2,3,4,6,5,4,3,2), & \beta_{2}=(2,2,3,4,3,2,1,0), \\
\beta_{3}=(0,1,1,2,2,2,1,0), & \beta_{4}=(0,1,1,2,1,0,0,0), \\
\beta_{5}=\alpha_{7}, \quad \beta_{6}=\alpha_{5}, & \beta_{7}=\alpha_{3}, \quad \beta_{8}=\alpha_{2} .
\end{aligned}
$$

Proposition 3.34. Let $x$ be an element of $E_{8}, p \neq 2$, and $\mathcal{O}$ its conjugacy class. If one of

(a) $x=h_{\alpha_{2}}(-1) h_{\alpha_{3}}(-1)$,

(b) $x=h_{\alpha_{2}}(-1) h_{\alpha_{5}}(-1) h_{\alpha_{7}}(-1) \sim h_{\alpha_{8}}(-1)$,

holds, then $\mathcal{O} \cap B w_{\mathcal{O}} B \cap B^{-}$is nonempty, $\operatorname{dim} \mathcal{O}=\ell\left(w_{\mathcal{O}}\right)+\operatorname{rk}\left(1-w_{\mathcal{O}}\right)$ and $\mathcal{O}$ is spherical. 
Proof. The group $E_{8}$, for $p \neq 2$, has 2 classes of involutions.

(a) Let $y=n_{\beta_{1}} \cdots n_{\beta_{8}} \in w_{0} B, w=s_{\beta_{1}} \cdots s_{\beta_{8}}=w_{0}$. Then

$$
y^{2}=h_{\beta_{1}}(-1) \cdots h_{\beta_{8}}(-1)=1,
$$

and $\operatorname{dim} \mathcal{O}_{y} \geq \operatorname{dim} B$ by Proposition 3.6. Since $C(x)$ is of type $D_{8}$, we conclude that $x \sim y$, so that $\mathcal{O} \cap B w_{0} B$ is nonempty, $\operatorname{dim} \mathcal{O}=\ell\left(w_{0}\right)+\operatorname{rk}\left(1-w_{0}\right)$ and $\mathcal{O}$ is spherical. As above, $\mathcal{O} \cap B w B \cap B^{-} \neq \varnothing$.

(b) Let $x=h_{\alpha_{8}}(-1)$, so that $C(x)$ is of type $E_{7} A_{1}$. Let

$$
g=n_{\beta_{1}} n_{\beta_{2}} n_{\beta_{3}} n_{\alpha_{7}} x_{\beta_{1}}(1) x_{\beta_{2}}(1) x_{\beta_{3}}(1) x_{\alpha_{7}}(1) .
$$

Then $g x g^{-1} \in \mathcal{O} \cap B w B \cap B^{-}$, with $w=s_{\beta_{1}} s_{\beta_{2}} s_{\beta_{3}} s_{\alpha_{7}}$ and $\operatorname{dim} \mathcal{O}=\ell(w)+\operatorname{rk}(1-w)$. We conclude by Proposition 3.6.

Proposition 3.35. Let $G=E_{8}$. The spherical (nontrivial) semisimple classes are represented by

$$
\begin{aligned}
& \text { none, } \quad \text { for } p=2 \text {, } \\
& \left.\begin{array}{l}
h_{\alpha_{2}}(-1) h_{\alpha_{3}}(-1), \\
h_{\alpha_{8}}(-1),
\end{array}\right\} \quad \text { for } p=3 \text { or } 5 \text {. }
\end{aligned}
$$

Proof. Let $x$ be a semisimple element of $G$, and assume $\mathcal{O}=\mathcal{O}_{x}$ is spherical. Without loss of generality $C(x)=L_{J}$, a pseudo-Levi subgroup of $G$. There exists a semisimple element $\tilde{x}$ in $G_{\mathbb{C}}$ such that $C(\tilde{x})$ is $L_{J}$ in $G_{\mathbb{C}}$. By Lemma 3.11 , it follows that $\mathcal{O}_{\tilde{x}}$ is a spherical semisimple conjugacy class in $G_{\mathbb{C}}$, and therefore, from the classification of semisimple spherical conjugacy classes in zero (or good odd) characteristic [Cantarini et al. 2005, Table 2; Carnovale 2010, Theorem 3.8], it follows that $L_{J}$ is of type $E_{7} A_{1}$ or $D_{8}$.

Let $p=2$. Then $Z\left(E_{7} A_{1}\right)=Z\left(D_{8}\right)=1$, so there are no nontrivial spherical semisimple classes.

For $p=3$ or 5, we conclude by Proposition 3.34.

We have established the information in Table 9, where $w_{\mathcal{O}}=w_{0} w_{J}$.

Proposition 3.36. Let $\mathcal{O}$ be the conjugacy class of a mixed element $g$ in $E_{8}, p=2$, 3 or 5 . Then $\mathcal{O}$ is not spherical.

\begin{tabular}{|c|cccc|}
\hline $\mathcal{O}$ & $J$ & $w_{\mathcal{O}}$ & $C(g)$ & $\operatorname{dim} \mathcal{O}$ \\
\hline$h_{\alpha_{8}}(-1)$ & $\{2,3,4,5\}$ & $s_{\beta_{1}} s_{\beta_{2}} s_{\beta_{3}} s_{\alpha_{7}}$ & $E_{7} A_{1}$ & 112 \\
\hline$h_{\alpha_{2}}(-1) h_{\alpha_{3}}(-1)$ & $\varnothing$ & $w_{0}$ & $D_{8}$ & 128 \\
\hline
\end{tabular}

Table 9. Spherical semisimple classes in $E_{8}, p=3$ or 5 . 
Proof. Let $g=s u$, the Jordan decomposition. Assume that $\mathcal{O}$ is spherical. Then both $\mathcal{O}_{s}$ and $\mathcal{O}_{u}$ are spherical, and therefore $C(s)$ is of type $E_{7} A_{1}$ or $A_{7}$. A dimensional argument rules out all the possibilities except the case that $C(s)$ is of type $E_{7} A_{1}$ and $u$ is a nonidentical unipotent element in the component $A_{1}$ of $C(s)$ (hence $p=3$ or 5). Therefore, without loss of generality we may assume $g=h_{\alpha_{8}}(-1) x_{\alpha_{8}}(1)$, which is a regular element of the standard Levi subgroup $L_{J}$, for $J=\{7,8\}$. By Lemma 3.14, there is an infinite family $\mathcal{F}=\left\{g_{m} \mid m \in k^{*}\right\} \subset L_{J}$ such that the $g_{m}$ are all $L_{J}$-conjugate (hence $G$-conjugate) to $g$, and pairwise not $B_{J}$-conjugate. By Lemma 3.10 the family $\left(B . g_{m}\right)_{m \in k^{*}}$ is an infinite family of (distinct) $B$-orbits, a contradiction. Hence $\mathcal{O}$ is not spherical.

Theorem 3.37. Let $G=E_{8}, p=2,3$ or 5. The spherical classes are either semisimple or unipotent. The semisimple classes are represented in Table 9, and the unipotent classes are represented in [Costantini 2012, Tables 10 and 11].

Type $\boldsymbol{F}_{4}$. We put

$$
\beta_{1}=(2,3,4,2), \quad \beta_{2}=(0,1,2,2), \quad \beta_{3}=(0,1,2,0), \quad \beta_{4}=(0,1,0,0) .
$$

Also, $\gamma_{1}$ is the highest short root $(1,2,3,2)$.

Proposition 3.38. Let $x$ be an element of $F_{4}, p \neq 2$, and $\mathcal{O}$ its conjugacy class. If one of

(a) $x=h_{\alpha_{2}}(-1) h_{\alpha_{4}}(-1) \sim h_{\alpha_{1}}(-1)$,

(b) $x=h_{\alpha_{4}}(-1)$,

holds, then $\mathcal{O} \cap B w_{\mathcal{O}} B \cap B^{-}$is nonempty, $\operatorname{dim} \mathcal{O}=\ell\left(w_{\mathcal{O}}\right)+\operatorname{rk}\left(1-w_{\mathcal{O}}\right)$ and $\mathcal{O}$ is spherical.

Proof. The group $F_{4}$, for $p \neq 2$, has 2 classes of involutions.

(a) Let $y=n_{\beta_{1}} \cdots n_{\beta_{4}} \in w_{0} B, w=s_{\beta_{1}} \cdots s_{\beta_{4}}=w_{0}$. Then

$$
y^{2}=h_{\beta_{1}}(-1) \cdots h_{\beta_{4}}(-1)=1 \text {, }
$$

and $\operatorname{dim} \mathcal{O}_{y} \geq \operatorname{dim} B$ by Proposition 3.6. Since $C(x)$ is of type $A_{1} C_{3}$, we conclude that $x \sim y$, so that $\mathcal{O} \cap B w_{0} B$ is nonempty, $\operatorname{dim} \mathcal{O}=\ell\left(w_{0}\right)+\operatorname{rk}\left(1-w_{0}\right)$ and $\mathcal{O}$ is spherical. As above, $\mathcal{O} \cap B w B \cap B^{-} \neq \varnothing$.

(b) We have $C(x)$ of type $B_{4}$. Let $g=n_{\gamma_{1}} x_{\gamma_{1}}(1)$. Then $g x g^{-1} \in \mathcal{O} \cap B w B \cap B^{-}$, with $w=s_{\gamma_{1}}$ and $\operatorname{dim} \mathcal{O}=\ell(w)+\operatorname{rk}(1-w)$. We conclude by Proposition 3.6.

Proposition 3.39. Let $G=F_{4}$. The spherical (nontrivial) semisimple classes are represented by

none, for $p=2$,

$$
\left.\begin{array}{l}
h_{\alpha_{1}}(-1), \\
h_{\alpha_{4}}(-1),
\end{array}\right\} \quad \text { for } p=3 \text {. }
$$




\begin{tabular}{|c|cccc|}
\hline $\mathcal{O}$ & $J$ & $w_{\mathcal{O}}$ & $C(g)$ & $\operatorname{dim} \mathcal{O}$ \\
\hline$h_{\alpha_{4}}(-1)$ & $\{1,2,3\}$ & $s_{\gamma_{1}}$ & $B_{4}$ & 16 \\
\hline$h_{\alpha_{1}}(-1)$ & $\varnothing$ & $w_{0}$ & $C_{3} A_{1}$ & 28 \\
\hline
\end{tabular}

Table 10. Spherical semisimple classes in $F_{4}, p=3$.

Proof. Let $x$ be a semisimple element of $G$, and assume $\mathcal{O}=\mathcal{O}_{x}$ is spherical. Without loss of generality $C(x)=L_{J}$, a pseudo-Levi subgroup of $G$. There exists a semisimple element $\tilde{x}$ in $G_{\mathbb{C}}$ such that $C(\tilde{x})$ is $L_{J}$ in $G_{\mathbb{C}}$. By Lemma 3.11, it follows that $\mathcal{O}_{\tilde{x}}$ is a spherical semisimple conjugacy class in $G_{\mathbb{C}}$, and therefore, from the classification of semisimple spherical conjugacy classes in zero (or good odd) characteristic [Cantarini et al. 2005, Table 2; Carnovale 2010, Theorem 3.9], it follows that $L_{J}$ is of type $C_{3} A_{1}$ or $B_{4}$.

Let $p=2$. Then $Z\left(C_{3} A_{1}\right)=Z\left(B_{4}\right)=1$, so there are no nontrivial spherical semisimple classes.

For $p=3$, we conclude by Proposition 3.38.

We have established the information in Table 10, where $w_{\mathcal{O}}=w_{0} w_{J}$.

We finally deal with mixed classes in $F_{4}$. We recall that over the complex numbers the principal model orbit is a mixed conjugacy class; see [Luna 2007, 3.3(6) and p. 300], and also [Costantini 2010, Table 24].

Proposition 3.40. Let $x=h_{\alpha_{4}}(-1) x_{\alpha_{1}}(1)$ in $F_{4}, p \neq 2$, and $\mathcal{O}$ its conjugacy class. Then $\mathcal{O} \cap B w_{\mathcal{O}} B \cap B^{-}$is nonempty, $\operatorname{dim} \mathcal{O}=\ell\left(w_{\mathcal{O}}\right)+\operatorname{rk}\left(1-w_{\mathcal{O}}\right)$ and $\mathcal{O}$ is spherical.

Proof. This is the mixed class in $F_{4}$ which is spherical in zero or good, odd characteristic. We can deal with this class with the same method used in the proof of [Cantarini et al. 2005, Theorem 23], and corrected in the proof of [Carnovale 2010, Theorem 3.9], to show that $B w_{0} B \cap \mathcal{O} \neq \varnothing$, so that $w_{\mathcal{O}}=w_{0}, \operatorname{dim} \mathcal{O}=$ $\ell\left(w_{0}\right)+\operatorname{rk}\left(1-w_{0}\right)$ and $\mathcal{O}$ is spherical. The (correct) argument at the end of the proof of [Cantarini et al. 2005, Theorem 23] shows that $\mathcal{O} \cap B w_{0} B \cap B^{-} \neq \varnothing$.

Proposition 3.41. Let $G=F_{4}$. The spherical mixed classes are represented by

$$
\begin{array}{ll}
\text { none, } & \text { for } p=2, \\
h_{\alpha_{4}}(-1) x_{\alpha_{1}}(1), & \text { for } p=3 .
\end{array}
$$

Proof. Let $g=s u$, the Jordan decomposition of a mixed element $g$. Assume that $\mathcal{O}=\mathcal{O}_{g}$ is spherical. Then both $\mathcal{O}_{s}$ and $\mathcal{O}_{u}$ are spherical, and therefore $C(s)$ is of type $C_{3} A_{1}$ or $B_{4}$. A dimensional argument rules out all the possibilities except the case that $C(s)$ is of type $B_{4}$ and $u$ is in the minimal unipotent class of $C(s)$ (hence $p=3)$. Therefore, without loss of generality we may assume $g=h_{\alpha_{4}}(-1) x_{\alpha_{1}}(1)$. We conclude by Proposition 3.40. 


\begin{tabular}{|c|ccc|}
\hline $\mathcal{O}$ & $J$ & $w_{\mathcal{O}}$ & $\operatorname{dim} \mathcal{O}$ \\
\hline$h_{\alpha_{4}}(-1) x_{\alpha_{1}}(1)$ & $\varnothing$ & $w_{0}$ & 28 \\
\hline
\end{tabular}

Table 11. Spherical mixed classes in $F_{4}, p=3$.

Theorem 3.42. Let $G=F_{4}, p=2$ or 3. If $p=2$, the spherical classes are unipotent and are represented in Table 13 of [Costantini 2012]. If $p=3$, the spherical semisimple classes are represented in Table 10, the spherical unipotent classes are represented in Table 12 of [Costantini 2012] and the spherical mixed classes are represented in Table 11.

Type $\boldsymbol{G}_{2}$. We put $\beta_{1}=(3,2)$ and $\beta_{2}=\alpha_{1}$. Also, $\gamma_{1}$ is the highest short root $(2,1)$. Proposition 3.43. Let $x$ be an element of $G_{2}$, any characteristic, and $\mathcal{O}$ its conjugacy class. If one of

(a) $x=h_{\alpha_{1}}(-1), p \neq 2$,

(b) $x=h_{\alpha_{1}}(\zeta), \zeta$ a primitive 3 rd root of $1, p \neq 3$,

holds, then $\mathcal{O} \cap B w_{\mathcal{O}} B \cap B^{-}$is nonempty, $\operatorname{dim} \mathcal{O}=\ell\left(w_{\mathcal{O}}\right)+\operatorname{rk}\left(1-w_{\mathcal{O}}\right)$ and $\mathcal{O}$ is spherical.

Proof. (a) For $p \neq 2$, the group $G_{2}$ has 1 class of involutions. Let

$$
y=n_{\beta_{1}} n_{\beta_{2}} \in w_{0} B, \quad w=s_{\beta_{1}} s_{\beta_{2}}=w_{0} .
$$

Then $y^{2}=h_{\beta_{1}}(-1) h_{\beta_{2}}(-1)=1$, and $\operatorname{dim} \mathcal{O}_{y} \geq \operatorname{dim} B$ by Proposition 3.6. We conclude that $x \sim y$, so that $\mathcal{O} \cap B w_{0} B$ is nonempty, $\operatorname{dim} \mathcal{O}=\ell\left(w_{0}\right)+\operatorname{rk}\left(1-w_{0}\right)$ and $\mathcal{O}$ is spherical. As above, $\mathcal{O} \cap B w B \cap B^{-} \neq \varnothing$.

(b) For $p \neq 3$, let $g=n_{\gamma_{1}} x_{\gamma_{1}}$ (1). Then $g x g^{-1} \in \mathcal{O} \cap B w B \cap B^{-}$, with $w=s_{\gamma_{1}}$ and $\operatorname{dim} \mathcal{O}=\ell(w)+\operatorname{rk}(1-w)$. We conclude by Proposition 3.6.

Proposition 3.44. Let $G=G_{2}$. The spherical semisimple classes are represented by

$$
\begin{array}{ll}
h_{\alpha_{1}}(-1), & \text { for } p=3, \\
h_{\alpha_{1}}(\zeta), \zeta \text { a primitive 3rd root of } 1, & \text { for } p=2 .
\end{array}
$$

Proof. Let $x$ be a semisimple element of $G$, and assume $\mathcal{O}=\mathcal{O}_{x}$ is spherical. Without loss of generality $C(x)=L_{J}$, a pseudo-Levi subgroup of $G$. There exists a semisimple element $\tilde{x}$ in $G_{\mathbb{C}}$ such that $C(\tilde{x})$ is $L_{J}$ in $G_{\mathbb{C}}$. By Lemma 3.11, it follows that $\mathcal{O}_{\tilde{x}}$ is a spherical semisimple conjugacy class in $G_{\mathbb{C}}$, and therefore, from the classification of semisimple spherical conjugacy classes in zero (or good odd) characteristic [Cantarini et al. 2005, Table 2; Carnovale 2010, Theorem 3.1], it follows that $L_{J}$ is of type $A_{1} \widetilde{A}_{1}$ or $A_{2}$. If $p=2$, then $Z\left(A_{1} \widetilde{A}_{1}\right)=1$. If $p=3$, then $Z\left(A_{2}\right)=1$ and we conclude by Proposition 3.43. 


\begin{tabular}{|c|cccc|}
\hline $\mathcal{O}$ & $J$ & $w_{\mathcal{O}}$ & $C(g)$ & $\operatorname{dim} \mathcal{O}$ \\
\hline $\begin{array}{c}h_{\alpha_{1}}(\zeta) \\
\zeta \text { a primitive 3rd root of 1 }\end{array}$ & $\{2\}$ & $s_{\gamma_{1}}$ & $A_{2}$ & 6 \\
\hline
\end{tabular}

Table 12. Spherical semisimple classes in $G_{2}, p=2$.

\begin{tabular}{|c|cccc|}
\hline $\mathcal{O}$ & $J$ & $w_{\mathcal{O}}$ & $C(g)$ & $\operatorname{dim} \mathcal{O}$ \\
\hline$h_{\alpha_{1}}(-1)$ & $\varnothing$ & $w_{0}$ & $A_{1} \widetilde{A}_{1}$ & 8 \\
\hline
\end{tabular}

Table 13. Spherical semisimple classes in $G_{2}, p=3$.

Theorem 3.45. Let $G=G_{2}, p=2,3$. The spherical classes are either semisimple or unipotent. The semisimple classes are represented in Tables 12 and 13, and the unipotent classes are represented in Tables 14 and 15 of [Costantini 2012].

Proof. By the above discussion, we are left to show that no mixed class is spherical. Let $g=s u$, the Jordan decomposition. Assume that $\mathcal{O}_{g}$ is spherical. Then both $\mathcal{O}_{s}$ and $\mathcal{O}_{u}$ are spherical, and therefore $C(s)$ is of type $A_{1} \widetilde{A}_{1}$ or $A_{2}$. A dimensional argument rules out all the possibilities.

\section{Final remarks}

Once we have achieved the classification of spherical conjugacy classes and proved that for every spherical conjugacy class $\mathcal{O}$ we have $\operatorname{dim} \mathcal{O}=\ell\left(w_{\mathcal{O}}\right)+\operatorname{rk}\left(1-w_{\mathcal{O}}\right)$, we can extend to all characteristics the results obtained in [Carnovale 2008; 2009; Cantarini et al. 2005; Lu 2011] for the zero and good odd characteristic cases. In [Cantarini et al. 2005, Theorem 25] we established the characterization of spherical conjugacy classes in terms of the dimension formula: a conjugacy class $\mathcal{O}$ in $G$ is spherical if and only if $\operatorname{dim} \mathcal{O}=\ell\left(w_{\mathcal{O}}\right)+\operatorname{rk}\left(1-w_{\mathcal{O}}\right)$. This was obtained over the complex numbers, and the same proof works over any algebraically closed field of characteristic zero. Then the same characterization was given in zero, or good odd characteristic in [Carnovale 2008, Theorem 4.4], without the classification of spherical conjugacy classes. Lu gave a very neat proof of the dimension formula (even for twisted conjugacy classes) in [Lu 2011, Theorem 1.1] in characteristic zero. From the results obtained in the previous section, we may state:

Theorem 4.1. Let $\mathcal{O}$ be a conjugacy class of a simple algebraic group, any characteristic. The following are equivalent:

(a) $\mathcal{O}$ is spherical;

(b) There exists $w \in W$ such that $\mathcal{O} \cap B w B \neq \varnothing$ and $\operatorname{dim} \mathcal{O} \leq \ell(w)+\operatorname{rk}(1-w)$;

(c) $\operatorname{dim} \mathcal{O}=\ell\left(w_{\mathcal{O}}\right)+\operatorname{rk}\left(1-w_{\mathcal{O}}\right)$. 
Corollary 4.2. Let $\mathcal{O}$ be a spherical class of $G$. Then $\operatorname{dim} \mathcal{O} \leq \ell\left(w_{0}\right)+\operatorname{rk}\left(1-w_{0}\right)$.

Proof. We have $\operatorname{dim} \mathcal{O}=\ell\left(w_{\mathcal{O}}\right)+\operatorname{rk}\left(1-w_{\mathcal{O}}\right)$, and

$$
\ell(w)+\operatorname{rk}(1-w) \leq \ell\left(w_{0}\right)+\operatorname{rk}\left(1-w_{0}\right)
$$

for every $w \in W$ (cf. [Carnovale 2008, Remark 4.14]).

Proposition 4.3. Let $\mathcal{O}$ be a spherical conjugacy class and $w=w_{\mathcal{O}}=w_{0} w_{J}$. Then $\left(T^{w}\right)^{\circ} \leq C_{T}(x) \leq T^{w}, C_{U}(x)=U_{w_{J}}$ and $\left(T^{w}\right)^{\circ} U_{w_{J}} \leq C_{B}(x) \leq T^{w} U_{w_{J}}$ for every $x \in \mathcal{O} \cap w B$.

Proof. We choose a representative $\dot{w}$ of $w$ in $N$ such that $x=\dot{w} u$ for $u \in U$. Let $b=t u_{1} u_{2} \in C_{B}(x)$, where $t \in T, u_{1} \in U_{w}$ and $u_{2} \in U_{w_{J}}$. From the Bruhat decomposition, we get $u_{1}=1$ and $t \in T^{w}$, so that $C_{B}(x) \leq T^{w} U_{w_{J}}$. But the dimension formula $\operatorname{dim} \mathcal{O}=\ell(w)+\operatorname{rk}(1-w)$ implies

$$
\operatorname{dim} C_{B}(x)=n-\operatorname{rk}(1-w)+\ell\left(w_{J}\right)=\operatorname{dim} T^{w} U_{w_{J}} .
$$

Hence $\left(C_{B}(x)\right)^{\circ}=\left(T^{w}\right)^{\circ} U_{w_{J}}$ and $C_{U}(x)=U_{w_{J}}$.

Now assume $b=t u_{1} \in C_{T U_{w}}(x)$, where $t \in T$ and $u_{1} \in U_{w}$. Again from the Bruhat decomposition, we get $u_{1}=1$ and $t \in T^{w}$, so that $C_{T U_{w}}(x)=C_{T}(x) \leq T^{w}$. We have $B \cdot x=T U_{w} U_{w_{J}} \cdot x=T U_{w} \cdot x$, hence $\operatorname{dim} C_{T U_{w}}(x)=n-\operatorname{rk}(1-w)=\operatorname{dim} T^{w}$. It follows that $\left(T^{w}\right)^{\circ} \leq C_{T}(x) \leq T^{w}$.

Theorem 4.4. Let $\mathcal{O}$ be a spherical conjugacy class of a simple algebraic group and $v=\mathcal{O} \cap B w_{\mathcal{O}} B$ the dense $B$-orbit. Then $C_{U}(x)$ is connected and $C_{B}(x)$ is a split extension of $\left(C_{B}(x)\right)^{\circ}$ by an elementary abelian 2-group for every $x \in v$. If $p=2$, then $C_{U}(x), C_{T}(x)$ and $C_{B}(x)$ are connected for every $x \in \mathcal{O} \cap B w_{\mathcal{O}} B$.

Proof. Let $w=w_{\mathcal{O}}$. We may assume $x \in w B$. From the discussion after [Costantini 2010, Corollary 3.22], we have $T=\left(T^{w}\right)^{\circ}\left(S^{w}\right)^{\circ}$, where $S^{w}=\left\{t \in T \mid t^{w}=t^{-1}\right\}$. Then $T^{w}=\left(T^{w}\right)^{\circ}\left(T^{w} \cap T_{2}\right)$, where $T_{2}=\left\{t \in T \mid t^{2}=1\right\}$, and $C_{T}(x)=\left(T^{w}\right)^{\circ} C_{T_{2}}(x)$ by Proposition 4.3. There exists a subgroup $R$ of $T_{2}$ such that $T^{w}=\left(T^{w}\right)^{\circ} \times R$, whence $C_{T}(x)=\left(T^{w}\right)^{\circ} \times C_{R}(x)$. In particular,

$$
C_{B}(x)=\left(\left(T^{w}\right)^{\circ} \times C_{R}(x)\right) U_{w_{J}}=\left(C_{B}(x)\right)^{\circ} C_{R}(x) .
$$

If $p=2$, then $T_{2}=\{1\}, T^{w}=\left(T^{w}\right)^{\circ}=C_{T}(x)$ and $C_{B}(x)=\left(C_{B}(x)\right)^{\circ}$.

We recall, from Remark 3.3, that there is an action of $W$ on the set $\mathcal{V}$ of $B$-orbits in $\mathcal{O}$ when $\mathcal{O}$ is a spherical conjugacy class and $p \neq 2$. We are now in the position to prove that this action is also defined for $p=2$.

Corollary 4.5. Let $\mathcal{O}$ be a spherical conjugacy class of a simple algebraic group, any characteristic. Then there is an action of the Weyl group $W$ on the set of $B$-orbits in $\mathcal{O}$ (as defined in [Knop 1995]). 
Proof. We have only to deal with $p=2$. By [Knop 1995, Theorem 4.2(c)], the action of $W$ is defined on the set of $B$-orbits in $\mathcal{O}$ as long as $C_{U}(x)$ is connected for every $x \in \mathcal{O}$. By Theorem 4.4, $C_{U}(x)$ is connected for every $x$ in the dense $B$-orbit; this ensures that $C_{U}(x)$ is connected for every $x \in \mathcal{O}$ by [Knop 1995, Corollary 3.4].

Once the $W$-action has been defined when $p=2$, we can extend to this case the results obtained by G. Carnovale in zero or good odd characteristic.

Theorem 4.6. Let $\mathcal{O}$ be a spherical conjugacy class of a simple algebraic group. If $\mathcal{O} \cap B w B$ is nonempty, then $w^{2}=1$.

Corollary 4.7. Let $\mathcal{O}$ be a spherical conjugacy class, and assume $\mathcal{O} \cap B w B \neq \varnothing$ for some $w \in W$. Then $\mathcal{O} \cap B z B \neq \varnothing$ for every conjugate $z$ of $w$ in $W$.

Theorem 4.8. Let $\mathcal{O}$ be a conjugacy class in a simple algebraic group. If

$$
\{w \in W \mid \mathcal{O} \cap B w B \neq \varnothing\} \subseteq\left\{w \in W \mid w^{2}=1\right\},
$$

then $\mathcal{O}$ is spherical.

Assume $\mathcal{O}$ is a spherical conjugacy class of a simple algebraic group (any characteristic), and $v$ the dense $B$-orbit in $\mathcal{O}$. Set $P=\{g \in G \mid g . v=v\}$. Then $P$ is a parabolic subgroup of $G$ containing $B$, and therefore $P=P_{K}$, the standard parabolic subgroup relative to a certain subset $K$ of $\Pi$.

Theorem 4.9. Let $\mathcal{O}$ be a spherical conjugacy class of a simple algebraic group, any characteristic, $w=w_{0} w_{J}$ be the unique element in $W$ such that $\mathcal{O} \cap B w B$ is dense in $\mathcal{O}, v=\mathcal{O} \cap B w B$ the dense B-orbit in $\mathcal{O}$ and $P_{K}=\{g \in G \mid g . v=v\}$. Then $K=J$. If $x \in \mathcal{O} \cap w B$, then $L_{J}^{\prime}$ and $\left(T^{w}\right)^{\circ}$ are contained in $C(x)$ and $C_{B}(x)^{\circ}=\left(T^{w}\right)^{\circ} U_{w_{J}}$.

Proof. We have already showed that $C_{B}(x)^{\circ}=\left(T^{w}\right)^{\circ} U_{w_{J}}$ for every $x \in \mathcal{O} \cap w B$. Let $S=\{i, \vartheta(i)\}$ be a $\vartheta$-orbit in $\Pi \backslash J$ consisting of 2 elements. We define $H_{S}=\left\{h_{\alpha_{i}}(z) h_{\alpha_{\vartheta}(i)}\left(z^{-1}\right) \mid z \in k^{*}\right\}$. Let $\mathcal{S}_{1}$ be the set of $\vartheta$-orbits in $\Pi \backslash J$ consisting of 2 elements. Then, by [Costantini 2010, Remark 3.10], $\Delta_{J} \cup\left\{\alpha_{i}-\alpha_{\vartheta(i)}\right\}_{\mathcal{S}_{1}}$ is a basis of $\operatorname{ker}(1-w)$ and

$$
\left(T^{w}\right)^{\circ}=\prod_{j \in J} H_{\alpha_{j}} \times \prod_{S \in \mathcal{S}_{1}} H_{S} .
$$

We put $\Psi_{J}=\{\beta \in \Phi \mid w(\beta)=-\beta\}$. Then $\Psi_{J}$ is a root system in $\operatorname{Im}(1-w)$ [Springer 1982, Proposition 2], and $\left.w\right|_{\operatorname{Im}(1-w)}$ is -1 . If $K=C\left(\left(T^{w}\right)^{\circ}\right)^{\prime}$, then $K$ is semisimple with root system $\Psi_{J}$ and maximal torus $T \cap K=\left(S^{w}\right)^{\circ}$. Assume $x=\dot{w} u \in v$, with $u \in U$. Then $\left(T^{w}\right)^{\circ} \leq C(x)$ implies $x \in C\left(\left(T^{w}\right)^{\circ}\right)$, and moreover, $\dot{w} \in C\left(T^{w}\right)$, so that $u \in K$. Let $u=\prod_{\alpha \in \Phi^{+} \cap \Psi_{J}} x_{\alpha}\left(k_{\alpha}\right)$ be the expression of $u$ for any fixed total ordering on $\Phi^{+}$. If $k_{\alpha} \neq 0$, then $w(\alpha)=-\alpha$, so that in particular $u \in U_{w}$. Moreover, if $\beta \in \Phi_{J}$, then $(\alpha, \beta)=(w \alpha, w \beta)=(-\alpha, \beta)$, so that $\alpha \perp \beta$. 
Finally, we have $\vartheta \alpha=-\alpha$, since $w \alpha=-\alpha$ is equivalent to $w_{J} \alpha=-w_{0} \alpha$, and $w_{J} \alpha=\alpha$, since $w_{J} \in W_{J}$ and $\left(\alpha, \alpha_{j}\right)=0$ for every $j \in J$.

From the fact that $U_{w_{J}} \leq C(x)$, it follows that $U_{w_{J}} \leq C(\dot{w})$, and therefore $U_{w_{J}} \leq C(u)$. From the Chevalley commutator formula, we deduce further that $w_{J} U_{w_{J}} w_{J}^{-1} \leq C(u)$, so that $L_{J}^{\prime} \leq C(x)$. Then we may argue as in the proof of [Carnovale 2008, Proposition 4.15] to conclude that $K=J$.

Remark 4.10. Assume $G$ is a connected reductive algebraic group over $k$. From the classification of spherical conjugacy classes obtained in simple algebraic groups (which is independent of the isogeny class), one gets the classification of spherical conjugacy classes in $G$. In fact, if $G=Z G_{1} \cdots G_{r}$, where $Z$ is the connected component of the center of $G$, and $G_{1}, \ldots, G_{r}$ are the simple components of $G$, then the conjugacy class $\mathcal{O}$ in $G$ of $x=z x_{1} \cdots x_{r}$, with $z \in Z$ and $x_{i} \in G_{i}$ for $i=1, \ldots, r$, is spherical if and only if the conjugacy class $\mathcal{O}_{i}$ of $x_{i}$ in $G_{i}$ is spherical for every $i=1, \ldots, r$.

Remark 4.11. In order to show that a conjugacy class $\mathcal{O}$ is spherical, we showed that $\operatorname{dim} \mathcal{O}=\ell\left(w_{\mathcal{O}}\right)+\operatorname{rk}\left(1-w_{\mathcal{O}}\right)$. However, in each case we even showed that $\mathcal{O} \cap B w_{\mathcal{O}} B \cap B^{-} \neq \varnothing$. The motivation for this was the proof of the De ConciniKac-Procesi conjecture for quantum groups at roots of one over spherical conjugacy classes; see [Cantarini et al. 2005]. The fact that $\mathcal{O} \cap B w_{\mathcal{O}} B \cap B^{-} \neq \varnothing$ for every spherical conjugacy class has been proved in characteristic zero in [Cantarini et al. 2005]. It is a general fact that if $\mathcal{O}$ is semisimple, then $\mathcal{O} \cap B w B \neq \varnothing$ implies $\mathcal{O} \cap B w B \cap B^{-} \neq \varnothing$ for any $w \in W$ [Cantarini et al. 2005, Lemma 14]. For unipotent classes, we showed in [Costantini 2012] for $p=2$ that $\mathcal{O} \cap B w_{\mathcal{O}} B \cap B^{-} \neq \varnothing$ by exhibiting explicitly an element in $\mathcal{O} \cap B w_{\mathcal{O}} B \cap B^{-}$. The argument in [Cantarini et al. 2005, Lemma 10] allows one to prove that $\mathcal{O} \cap B w_{\mathcal{O}} B \cap B^{-} \neq \varnothing$ for every spherical unipotent class in good characteristic. However, it is possible to adapt the same proof to the remaining unipotent classes in bad characteristic, due to the fact that we do have the classification, and so we just make a case by case consideration. Assume $\mathcal{O}$ is a spherical mixed class. In all cases, apart from $F_{4}$, we have an explicit element in $\mathcal{O} \cap B w_{\mathcal{O}} B \cap B^{-}$. We observed in Proposition 3.40 that the argument used in [Cantarini et al. 2005] holds for every odd characteristic. We conclude that in all characteristics, if $\mathcal{O}$ is a spherical conjugacy class, then $\mathcal{O} \cap B w_{\mathcal{O}} B \cap B^{-} \neq \varnothing$.

\section{References}

[Bourbaki 1981] N. Bourbaki, Éléments de mathématique: algèbre, chapitres 4 à 7, 2ème ed., Masson, Paris, 1981. MR Zbl

[Brion 1986] M. Brion, "Quelques propriétés des espaces homogènes sphériques”, Manuscripta Math. 55:2 (1986), 191-198. MR Zbl

[Brion 2001] M. Brion, "On orbit closures of spherical subgroups in flag varieties", Comment. Math. Helv. 76:2 (2001), 263-299. MR Zbl 
[Brundan 1998] J. Brundan, "Dense orbits and double cosets", pp. 259-274 in Algebraic groups and their representations (Cambridge, 1997), edited by R. W. Carter and J. Saxl, NATO Adv. Sci. Inst. Ser. C Math. Phys. Sci. 517, Kluwer Acad. Publ., Dordrecht, 1998. MR Zbl

[Cantarini et al. 2005] N. Cantarini, G. Carnovale, and M. Costantini, "Spherical orbits and representations of $U_{\epsilon}(\mathfrak{g})$ )", Transform. Groups 10:1 (2005), 29-62. MR Zbl

[Carnovale 2008] G. Carnovale, "Spherical conjugacy classes and involutions in the Weyl group", Math. Z. 260:1 (2008), 1-23. MR Zbl

[Carnovale 2009] G. Carnovale, "Spherical conjugacy classes and the Bruhat decomposition", Ann. Inst. Fourier (Grenoble) 59:6 (2009), 2329-2357. MR Zbl

[Carnovale 2010] G. Carnovale, "A classification of spherical conjugacy classes in good characteristic", Pacific J. Math. 245:1 (2010), 25-45. MR Zbl

[Carnovale and Costantini 2013] G. Carnovale and M. Costantini, "On Lusztig's map for spherical unipotent conjugacy classes”, Bull. Lond. Math. Soc. 45:6 (2013), 1163-1170. MR Zbl

[Carter 1985] R. W. Carter, Finite groups of Lie type: conjugacy classes and complex characters, Wiley, New York, 1985. MR Zbl

[Carter 1989] R. W. Carter, Simple groups of Lie type, Wiley, New York, 1989. MR Zbl

[Chan et al. 2010] K. Y. Chan, J.-H. Lu, and S. K.-M. To, "On intersections of conjugacy classes and Bruhat cells", Transform. Groups 15:2 (2010), 243-260. MR Zbl

[Costantini 2010] M. Costantini, "On the coordinate ring of spherical conjugacy classes", Math. Z. 264:2 (2010), 327-359. MR Zbl

[Costantini 2012] M. Costantini, "A classification of unipotent spherical conjugacy classes in bad characteristic”, Trans. Amer. Math. Soc. 364:4 (2012), 1997-2019. MR Zbl

[Fowler and Röhrle 2008] R. Fowler and G. Röhrle, "Spherical nilpotent orbits in positive characteristic", Pacific J. Math. 237:2 (2008), 241-286. MR Zbl

[Grosshans 1992] F. D. Grosshans, "Contractions of the actions of reductive algebraic groups in arbitrary characteristic”, Invent. Math. 107:1 (1992), 127-133. MR Zbl

[Humphreys 1975] J. E. Humphreys, Linear algebraic groups, Graduate Texts in Mathematics 21, Springer, New York, 1975. MR Zbl

[Iwahori 1970] N. Iwahori, "Centralizers of involutions in finite Chevalley groups”, pp. 267-295 in Seminar on algebraic groups and related finite groups (Princeton, 1968), Lecture Notes in Mathematics 131, Springer, Berlin, 1970. MR Zbl

[Knop 1995] F. Knop, "On the set of orbits for a Borel subgroup", Comment. Math. Helv. 70:2 (1995), 285-309. MR Zbl

[Lu 2011] J.-H. Lu, "On a dimension formula for spherical twisted conjugacy classes in semisimple algebraic groups”, Math. Z. 269:3-4 (2011), 1181-1188. MR Zbl

[Luna 2007] D. Luna, "La variété magnifique modèle", J. Algebra 313:1 (2007), 292-319. MR Zbl

[Mars and Springer 1998] J. G. M. Mars and T. A. Springer, "Hecke algebra representations related to spherical varieties", Represent. Theory 2 (1998), 33-69. MR Zbl

[McNinch and Sommers 2003] G. J. McNinch and E. Sommers, "Component groups of unipotent centralizers in good characteristic", J. Algebra 260:1 (2003), 323-337. MR Zbl

[Panyushev 1994] D. I. Panyushev, "Complexity and nilpotent orbits", Manuscripta Math. 83:3-4 (1994), 223-237. MR Zbl

[Panyushev 1999] D. I. Panyushev, "On spherical nilpotent orbits and beyond", Ann. Inst. Fourier (Grenoble) 49:5 (1999), 1453-1476. MR Zbl 
[Perkins and Rowley 2002] S. B. Perkins and P. J. Rowley, "Minimal and maximal length involutions in finite Coxeter groups", Comm. Algebra 30:3 (2002), 1273-1292. MR Zbl

[Richardson and Springer 1990] R. W. Richardson and T. A. Springer, "The Bruhat order on symmetric varieties", Geom. Dedicata 35:1-3 (1990), 389-436. MR Zbl

[Sommers 1998] E. Sommers, "A generalization of the Bala-Carter theorem for nilpotent orbits", Internat. Math. Res. Notices 11 (1998), 539-562. MR Zbl

[Spaltenstein 1982] N. Spaltenstein, Classes unipotentes et sous-groupes de Borel, Lecture Notes in Mathematics 946, Springer, Berlin, 1982. MR Zbl

[Springer 1982] T. A. Springer, "Some remarks on involutions in Coxeter groups", Comm. Algebra 10:6 (1982), 631-636. MR Zbl

[Springer 1998a] T. A. Springer, Linear algebraic groups, 2nd ed., Progress in Mathematics 9, Birkhäuser, Boston, 1998. MR Zbl

[Springer 1998b] T. A. Springer, "Schubert varieties and generalizations", pp. 413-440 in Representation theories and algebraic geometry (Montreal, 1997), edited by A. Broer and G. Sabidussi, NATO Adv. Sci. Inst. Ser. C Math. Phys. Sci. 514, Kluwer Acad. Publ., Dordrecht, 1998. MR Zbl

[Steinberg 1968] R. Steinberg, Lectures on Chevalley groups, Yale University, New Haven, CT, 1968. MR Zbl

[Vinberg 1986] È. B. Vinberg, "Complexity of actions of reductive groups", Funct. Anal. Appl. 20:1 (1986), 1-13. MR Zbl

Received April 21, 2015. Revised April 14, 2016.

Mauro Costantini

DiPARTIMENTO DI MATEMATICA

UNIVERSITÀ DEGLI STUDI DI PADOVA

VIA TRIESTE 63

35121 PADOVA

ITALY

costantini@math.unipd.it 


\title{
PACIFIC JOURNAL OF MATHEMATICS
}

Founded in 1951 by E. F. Beckenbach (1906-1982) and F. Wolf (1904-1989)

$$
\text { msp.org/pjm }
$$

\section{EDITORS}

\author{
Don Blasius (Managing Editor) \\ Department of Mathematics \\ University of California \\ Los Angeles, CA 90095-1555 \\ blasius@math.ucla.edu
}

\author{
Paul Balmer \\ Department of Mathematics \\ University of California \\ Los Angeles, CA 90095-1555 \\ balmer@math.ucla.edu \\ Robert Finn \\ Department of Mathematics \\ Stanford University \\ Stanford, CA 94305-2125 \\ finn@math.stanford.edu \\ Sorin Popa \\ Department of Mathematics \\ University of California \\ Los Angeles, CA 90095-1555 \\ popa@math.ucla.edu
}

\author{
Vyjayanthi Chari \\ Department of Mathematics \\ University of California \\ Riverside, CA 92521-0135 \\ chari@math.ucr.edu \\ Kefeng Liu \\ Department of Mathematics \\ University of California \\ Los Angeles, CA 90095-1555 \\ liu@math.ucla.edu \\ Igor Pak \\ Department of Mathematics \\ University of California \\ Los Angeles, CA 90095-1555 \\ pak.pjm@gmail.com \\ Paul Yang \\ Department of Mathematics \\ Princeton University \\ Princeton NJ 08544-1000 \\ yang@math.princeton.edu
}

\section{PRODUCTION}

Silvio Levy, Scientific Editor, production@msp.org

\section{SUPPORTING INSTITUTIONS}

ACADEMIA SINICA, TAIPEI

CALIFORNIA INST. OF TECHNOLOGY

STANFORD UNIVERSITY

UNIV. OF BRITISH COLUMBIA

UNIV. OF CALIFORNIA, BERKELEY

UNIV. OF CALIFORNIA, DAVIS

UNIV. OF CALIFORNIA, LOS ANGELES

UNIV. OF CALIFORNIA, RIVERSIDE

UNIV. OF CALIFORNIA, SAN DIEGO

UNIV. OF CALIF., SANTA BARBARA
KEIO UNIVERSITY

MATH. SCIENCES RESEARCH INSTITUTE

NEW MEXICO STATE UNIV.

OREGON STATE UNIV.
Daryl Cooper

Department of Mathematics

University of California

Santa Barbara, CA 93106-3080 cooper@math.ucsb.edu

Jiang-Hua Lu

Department of Mathematics

The University of Hong Kong

Pokfulam Rd., Hong Kong

jhlu@maths.hku.hk

$$
\text { Jie Qing }
$$

Department of Mathematics

University of California

Santa Cruz, CA 95064

qing@ cats.ucsc.edu

\author{
UNIV. OF CALIF., SANTA CRUZ \\ UNIV. OF MONTANA \\ UNIV. OF OREGON \\ UNIV. OF SOUTHERN CALIFORNIA \\ UNIV. OF UTAH \\ UNIV. OF WASHINGTON \\ WASHINGTON STATE UNIVERSITY
}

These supporting institutions contribute to the cost of publication of this Journal, but they are not owners or publishers and have no responsibility for its contents or policies.

See inside back cover or msp.org/pjm for submission instructions.

The subscription price for 2016 is US $\$ 440 /$ year for the electronic version, and \$600/year for print and electronic.

Subscriptions, requests for back issues and changes of subscriber address should be sent to Pacific Journal of Mathematics, P.O. Box 4163, Berkeley, CA 94704-0163, U.S.A. The Pacific Journal of Mathematics is indexed by Mathematical Reviews, Zentralblatt MATH, PASCAL CNRS Index, Referativnyi Zhurnal, Current Mathematical Publications and Web of Knowledge (Science Citation Index).

The Pacific Journal of Mathematics (ISSN 0030-8730) at the University of California, c/o Department of Mathematics, 798 Evans Hall \#3840, Berkeley, CA 94720-3840, is published twelve times a year. Periodical rate postage paid at Berkeley, CA 94704, and additional mailing offices. POSTMASTER: send address changes to Pacific Journal of Mathematics, P.O. Box 4163, Berkeley, CA 94704-0163.

PJM peer review and production are managed by EditFLOW ${ }^{\circledR}$ from Mathematical Sciences Publishers.

PUBLISHED BY

\section{I. mathematical sciences publishers}

nonprofit scientific publishing

http://msp.org/

(C) 2016 Mathematical Sciences Publishers 


\section{PACIFIC JOURNAL OF MATHEMATICS}

Volume $285 \quad$ No. $1 \quad$ November 2016

Iwahori-Hecke algebras for Kac-Moody groups over local fields

Nicole BARDY-PANSE, StÉPHANE GAUSSENT and GuY

ROUSSEAU

A classification of spherical conjugacy classes

MAURo Costantini

Affine weakly regular tensor triangulated categories

IVo DELL'AMBROGIO and DONALD STANLEY

Involutive automorphisms of $N_{\circ}^{\circ}$-groups of finite Morley rank

ADRIEN DELORO and ÉRIC JALIGOT

Schur-Weyl duality for Deligne categories, II: The limit case

INNA ENTOVA AIZENBUD

A generalization of the Greene-Krantz theorem for the semicontinuity 225 property of automorphism groups

JAE-CHEON JOO

Gradient estimates for a nonlinear Lichnerowicz equation under general geometric flow on complete noncompact manifolds

LIANG ZHAO and SHOUWEN FANG 